\title{
MEROMORPHIC EXTENSION OF ANALYTIC CONTINUED FRACTIONS ACROSS THEIR DIVERGENCE LINE WITH APPLICATIONS TO ORTHOGONAL POLYNOMIALS
}

\author{
HANS-J. RUNCKEL
}

\begin{abstract}
For the limit periodic $J$-fraction $K\left(-a_{n} /\left(\lambda+b_{n}\right)\right), a_{n}, b_{n} \in \mathbb{C}$, $n \in \mathbb{N}$, which is normalized such that it converges and represents a meromorphic function $f(\lambda)$ on $\mathbb{C}^{*}:=\mathbb{C} \backslash[-1,1]$, the numerators $A_{n}$ and denominators $B_{n}$ of its $n$th approximant are explicitly determined for all $n \in \mathbb{N}$. Under natural conditions on the speed of convergence of $a_{n}, b_{n}, n \rightarrow \infty$, the asymptotic behaviour of the orthogonal polynomials $B_{n}, A_{n+1}$ (of first and second kind) is investigated on $\mathbb{C}^{*}$ and $[-1,1]$. An explicit representation for $f(\lambda)$ yields continuous extension of $f$ from $\mathbb{C}^{*}$ onto upper and lower boundary of the cut $(-1,1)$. Using this and a determinant relation, which asymptotically connects both sequences $A_{n}, B_{n}$, one obtains nontrivial explicit formulas for the absolutely continuous part (weight function) of the distribution functions for the orthogonal polynomial sequences $B_{n}, A_{n+1}, n \in \mathbb{N}$. This leads to short proofs of results which generalize and supplement results obtained by P. G. Nevai [7]. Under a stronger condition the explicit representation for $f(\lambda)$ yields meromorphic extension of $f$ from $\mathbb{C}^{*}$ across $(-1,1)$ onto a region of a second copy of $\mathbb{C}$ which there is bounded by an ellipse, whose focal points \pm 1 are first order algebraic branch points for $f$. Then, by substitution, analogous results on continuous and meromorphic extension are obtained for limit periodic continued fractions $K\left(-a_{n}(z) /\left(\lambda(z)+b_{n}(z)\right)\right)$, where $a_{n}(z), b_{n}(z), \lambda(z)$ are holomorphic on a region in $\mathbb{C}$. Finally, for $T$-fractions $T(z)=K\left(-c_{n} z /\left(1+d_{n} z\right)\right)$ with $c_{n} \rightarrow c, d_{n} \rightarrow d, n \rightarrow \infty$, the exact convergence regions are determined for all $c, d \in \mathbb{C}$. Again, explicit representations for $T(z)$ yield continuous and meromorphic extension results. For all $c, d \in \mathbb{C}$ the regions (on Riemann surfaces) onto which $T(z)$ can be extended meromorphically, are described explicitly.
\end{abstract}

\section{INTRODUCTION AND BASIC NOTATIONS}

The main goal of this paper is to describe a general method of analytic continuation of meromorphic functions beyond the region in which they are defined by a convergent limit periodic analytic continued fraction. We first consider limit

Received by the editors August 23, 1989 and, in revised form, September 28, 1990.

1980 Mathematics Subject Classification (1985 Revision). Primary 30B70, 39A10, 40A15, 42C05, 30B40; Secondary 30E15, 30E20.

Key words and phrases. Limit periodic analytic continued fraction, $J$-fraction, $T$-fraction, meromorphic extension, continuous extension onto boundary, asymptotic behaviour and weight function of orthogonal polynomials, explicit solution of second order linear difference equations. 
periodic analytic continued fractions of the following special type ( $J$-fraction)

$$
f(\lambda)=\frac{1}{\lambda+b_{0}}-\frac{a_{1}}{\lambda+b_{1}}-\frac{a_{2}}{\lambda+b_{2}}-\cdots,
$$

where $a_{n}, b_{n-1} \in \mathbb{C}, a_{n} \neq 0$ for $n \in \mathbb{N}$ and $\lim _{n \rightarrow \infty} a_{n}=1 / 4, \lim _{n \rightarrow \infty} b_{n}=0$. If, more generally, $\lim _{n \rightarrow \infty} a_{n}=a \neq 0$ holds, then a suitable equivalence transformation and variable substitution reduces (1) to the case $a=1 / 4$. As usual we put for $n \geq 1 \quad\left(a_{0}:=1\right)$,

$$
A_{n}(\lambda) / B_{n}(\lambda):=\frac{1}{\lambda+b_{0}}-\frac{a_{1}}{\lambda+b_{1}}-\frac{a_{2}}{\lambda+b_{2}}-\cdots-\frac{a_{n-1}}{\lambda+b_{n-1}},
$$

where $A_{n}, B_{n}$ are polynomials in $\lambda$ of degree $n-1$ and $n$ respectively, which for $n \geq 1$ satisfy

$$
\left\{\begin{array}{lll}
A_{n+1}=\left(\lambda+b_{n}\right) A_{n}-a_{n} A_{n-1}, & A_{0}=0, & A_{1}=1, \\
B_{n+1}=\left(\lambda+b_{n}\right) B_{n}-a_{n} B_{n-1}, & B_{0}=1, & B_{1}=\lambda+b_{0} .
\end{array}\right.
$$

It is well known (see $[6,8,15])$, that under the above conditions the right side in (1) converges and represents a meromorphic function

$$
f(\lambda):=\lim _{n \rightarrow \infty} A_{n}(\lambda) / B_{n}(\lambda)
$$

in $\mathbb{C}^{*}:=\mathbb{C} \backslash[-1,1]$ with $f(\infty)=0$. In this paper we assume that (1) satisfies the additional condition

$$
\sum_{j=1}^{\infty}\left(\left|a_{j}-1 / 4\right|+\left|b_{j}\right|\right) R^{j}<\infty \text { for some } R \geq 1 .
$$

Sometimes we also will use the following condition

$$
\sum_{j=1}^{\infty} j\left(\left|a_{j}-1 / 4\right|+\left|b_{j}\right|\right)<\infty
$$

Now the substitution $\lambda=\Lambda(\omega):=\left(\omega+\omega^{-1}\right) / 2, \omega \in \mathbb{C}, \omega \neq 0$, maps $0<|\omega|<1$ as well as $|\omega|>1$ conformally onto $\mathbb{C}^{*}$ and $|\omega|=1$ bijectively onto both (upper and lower) boundaries of the cut $[-1,1]$. We define as inverse function of $\Lambda(\omega)$,

$$
\omega=\omega(\lambda):=\lambda-\left(\lambda^{2}-1\right)^{1 / 2}=\left((\lambda+1)^{1 / 2}-(\lambda-1)^{1 / 2}\right)^{2} / 2, \quad \lambda \in \mathbb{C}^{*},
$$

where the roots are chosen to be $>0$ for $\lambda>1$. Then $|\omega(\lambda)|<1$ holds for $\lambda \in \mathbb{C}^{*}$. Let $\mathbb{C}^{* *}$ be the complete 2 -sheeted Riemann surface obtained by analytic extension of $\omega(\lambda)$ from $\mathbb{C}^{*}$ across $[-1,1]$ into a second copy of $\mathbb{C}$. Then always $|\omega(\lambda)|>1$ holds in the second copy of $\mathbb{C} \backslash[-1,1]$.

In $\S 2$, Lemma 1, explicit formulas for $A_{n}(\Lambda(\omega)), B_{n}(\Lambda(\omega))$ are derived in terms of $\omega$, which together with (5) yield an explicit solution of the difference equation (2). Under the conditions (3) or (4) the asymptotic behaviour of $A_{n}, B_{n}$, as $n \rightarrow \infty$, via (14) is determined in Corollaries 1 and 2 and Theorem 5. In $\S 3$, Theorems 1 and 2 , the main results concerning continued fractions of type (1) are presented. If (3) holds with $R=1$, then the results of $\S 2$ lead to explicit series representations of holomorphic functions $A(\lambda), B(\lambda)$ such that for $\lambda \in \mathbb{C}^{*},(1)$ converges to $f(\lambda)=A(\lambda) / B(\lambda)$. For each $\lambda \in(-1,1)$ the asymptotic behaviour of $A_{n}(\lambda) / B_{n}(\lambda), n \rightarrow \infty$, explicitly reveals the divergence 
of (1). If (3) holds for some $R>1$, then $A(\lambda), B(\lambda)$ yield the meromorphic extension of $f(\lambda)$ from $\mathbb{C}^{*}$ across $[-1,1]$ into a subregion of the Riemann surface $\mathbb{C}^{* *}$ of $\omega(\lambda)$ which is bounded by the ellipse $|\omega(\lambda)|=R^{1 / 2}$, or explicitly

(6) $E(R):=\left\{\lambda \in \mathbb{C}:\left(\operatorname{Re} \lambda /\left(R^{1 / 2}+R^{-1 / 2}\right)\right)^{2}+\left(\operatorname{Im} \lambda /\left(R^{1 / 2}-R^{-1 / 2}\right)\right)^{2}=1 / 4\right\}$,

whose focal points \pm 1 are algebraic branch points of order 1 for $f(\lambda)$. If, in particular, (3) holds for all $R>1$, then $f(\lambda)$ can be extended meromorphically onto the complete Riemann surface $\mathbb{C}^{* *}$.

In $\S 4$, Theorems $3-5, B_{n}(\lambda)$, and $A_{n+1}(\lambda), n \geq 0$, are considered as orthogonal polynomial sequences of first and second kind. Under the conditions (3) with $R=1$ or (4) their asymptotic behaviour, as $n \rightarrow \infty$, on $\mathbb{C}^{*}$ and on $[-1,1]$ as well as generating functions are derived from the results of $\S 2$. The behaviour on $[-1,1]$ essentially depends on the fact that $A(\lambda), B(\lambda)$ can be extended continuously from $\mathbb{C}^{*}$ onto upper and lower boundary of $(-1,1)$. Applying the asymptotic formulas to a Wronskian-type relation which connects both sequences $A_{n}, B_{n}$ one obtains the identity (52) in Theorem 1. This identity connects an integral representation for $f(\lambda), \lambda \in \mathbb{C}^{*}$, with nontrivial explicit representations for the absolutely continuous parts of the distribution functions for both orthogonal polynomial sequences $B_{n}(\lambda), A_{n+1}(\lambda), n \geq 0$. The results of Theorems 3-5 generalize and complement results of $P$. G. Nevai [7] on orthogonal polynomials with real $a_{n}, b_{n}$.

In $\S 5$ general limit periodic analytic continued fractions

$$
F(z)=\frac{1}{\lambda(z)+b_{0}(z)}-\frac{a_{1}(z)}{\lambda(z)+b_{1}(z)}-\frac{a_{2}(z)}{\lambda(z)+b_{2}(z)}-\cdots
$$

are considered, where we assume that $a_{n}(z) \not \equiv 0, b_{n-1}(z), n \geq 1$, and $\lambda(z)$ are holomorphic functions of $z$ on a region $G \subset \mathbb{C}$, such that $\lim _{n \rightarrow \infty} b_{n}(z) \equiv \theta$ and $\lim _{n \rightarrow \infty} a_{n}(z) \equiv 1 / 4$ hold uniformly on each compact subset of $G$. If, more generally, $\lim _{n \rightarrow \infty} a_{n}(z)=a(z) / 4 \not \equiv 0$ holds on $G$, then an equivalence transformation applied to (7) yields

$$
F(z)=\frac{a^{-1 / 2}}{a^{-1 / 2} \lambda+a^{-1 / 2} b_{0}}-\frac{a^{-1} a_{1}}{a^{-1 / 2} \lambda+a^{-1 / 2} b_{1}}-\frac{a^{-1} a_{2}}{a^{-1 / 2} \lambda+a^{-1 / 2} b_{2}}-\cdots
$$

which, except for the first partial numerator $a^{-1 / 2}$, is a continued fraction of type (7), its value being independent of the chosen branch of $a^{-1 / 2}$. Of course, now $\lim _{n \rightarrow \infty} a^{-1} a_{n} \equiv 1 / 4$ holds uniformly on compact subsets of $G \backslash\{$ zeros of $a(z)\}$.

We now define the open set

$$
G^{*}:=G \backslash S, \quad \text { where } S:=\{z \in G: \lambda(z) \in[-1,1]\} .
$$

Here $G^{*}=\varnothing$ holds iff $\lambda(z)$ is a constant $\in[-1,1]$. We always assume $G^{*} \neq \varnothing$. Then $G^{*}$ is an at most countable union of disjoint regions $\neq \varnothing$, the components of $G^{*}$. Under the above conditions on each component of $G^{*}$ the right side in (7) converges and represents a meromorphic function $F(z)$ or tends to $\infty$. In analogy with (3) we assume in this paper that (7) satisfies the condition

$\sum_{j=1}^{\infty}\left(\left|a_{j}(z)-1 / 4\right|+\left|b_{j}(z)\right|\right) R^{j}<\infty$ uniformly on each compact subset of $G$ for some fixed $R \geq 1$. 
Sometimes we also will use the following condition

$$
\begin{aligned}
& \sum_{j=1}^{\infty} j\left(\left|a_{j}(z)-1 / 4\right|+\left|b_{j}(z)\right|\right)<\infty \text { uniformly on each compact } \\
& \text { subset of } G \text {. }
\end{aligned}
$$

On each component of $G^{*}$ we define (see (5))

$$
\hat{\omega}(z):=\omega(\lambda(z))=\lambda(z)-\left(\lambda^{2}(z)-1\right)^{1 / 2},
$$

where again $\left(\lambda^{2}-1\right)^{1 / 2}$ is chosen to be $>0$ for $\lambda>1, \lambda \in \mathbb{C}^{*}$. Then $0<|\hat{\omega}(z)|<1$ holds on each component of $G^{*}$. Let $G^{* *}$ denote the 2-sheeted Riemann surface of $\hat{\omega}(z)$ over $G$, obtained by analytic extension of $\hat{\omega}$ from each component of $G^{*}$ across the cut $S$ into a second copy of $G$. Thus, whenever $z$ crosses $S, \hat{\omega}(z)$ crosses $|\omega|=1$.

Then in Theorems 6 and 7, the main results concerning continued fractions of type (7), $F(z)$ is represented as $\widehat{A}(z) / \widehat{B}(z)$, where the holomorphic functions $\widehat{A}, \widehat{B}$ are obtained from the explicit series representations for $A(\lambda), B(\lambda)$ in $\S 3$ by substituting $a_{j}(z), b_{j}(z), \lambda(z)$ for $a_{j}, b_{j}, \lambda$. In analogy with the results of $\S 3$ one obtains detailed information concerning convergence of $(7)$ on $G^{*}$, divergence on $S$ and meromorphic extension of $F(z)$ from $G^{*}$ across $S$ into $G^{* *}$. If, in particular, (9) holds for all $R>1$, then $F(z)$ can be extended meromorphically from each component of $G^{*}$ on which $\widehat{B} \not \equiv 0$ onto the complete Riemann surface $G^{* *}$. An even more far-reaching main result is obtained in Theorem 8.

Finally, Theorems 6 and 7 will be applied in $\S 6$ to general limit periodic $T$-fractions

$$
T(z)=\frac{1}{1+d_{0} z}-\frac{c_{1} z}{1+d_{1} z}-\frac{c_{2} z}{1+d_{2} z}-\cdots,
$$

where $c_{n}, d_{n-1} \in \mathbb{C}, c_{n} \neq 0, n \geq 1, \lim _{n \rightarrow \infty} c_{n}=: c \in \mathbb{C}$ and $\lim _{n \rightarrow \infty} d_{n}=$ : $d \in \mathbb{C}$, such that

$$
\sum_{j=1}^{\infty}\left(\left|c_{j}-c\right|+\left|d_{j}-d\right|\right) R^{j}<\infty \text { holds for some } R \geq 1 .
$$

In Theorems 9 and 10, the main results concerning continued fractions of type (12), all possible combinations of $c, d \in \mathbb{C}$ are reduced to 6 special cases. In all these cases the divergence line $S$ of (12) and, if (13) holds for some $R>1$, the boundary curves $S(R)$ of regions into which meromorphic extension of $T(z)$ across $S$ is possible are described explicitly.

The method of meromorphic extension developed in the present paper generalizes and also simplifies a method used by the author in $[9,11]$ in the special case of (1), where all $b_{n}=0$ and in the special case of (12) where all $d_{n}=0$.

Another method of meromorphic extension which is entirely different from the one used in the present paper is the general method of "modified" continued fractions developed and investigated in the work of Gill [3], Jacobsen [4, 5], Thron and Waadeland [13, 14].

It seems that, under the assumption (13) for some $R>1$, this method so far has only been applied in [14] to the special case of $T$-fractions (12) where $c=d=1$, the result obtained there being identical with the one derived in Theorem 10 below, and to the case, where all $d_{n}=0$ and $c \neq 0$, when (12) is a regular $C$-fraction. In this case an explicit formula for the boundary curve 
$S(R)$ was already given in [9] and is again derived in Theorem 10 for the general case $d=0, c \neq 0$. It seems that the same curve $S(R)$ is obtained in [14] but in an implicit form.

In his Ph.D. thesis [12] Schlierf has applied the method of "modified" continued fractions, under the assumption (13) for some $R>1$, to other cases of (12) (essentially $d=1$ and $c \in \mathbb{R}$ ). These results turned out to be not as far-reaching and explicit as the corresponding results of Theorem 10 below. Even in the special case when (13) holds for all $R>1$, this method only yields meromorphic extension of $T(z)$ across $S$ into a proper subregion of the complete Riemann surface onto which extension then actually is possible according to Theorem 10.

\section{FURTHER NOTATIONS, AUXILIARY FORMULAS AND MAIN LEMMAS}

In order to determine $A_{n}, B_{n}$ in (2) explicitly for all $n$ we use the substitution $\lambda=\Lambda(\omega)=\left(\omega+\omega^{-1}\right) / 2$ and define for $n \geq 0, \omega \in \mathbb{C}$,

$$
\left\{\begin{array}{l}
C_{n}=C_{n}(\omega):=\left(1-\omega^{2}\right)(2 \omega)^{n-1} A_{n}(\Lambda(\omega)), \\
D_{n}=D_{n}(\omega):=\left(1-\omega^{2}\right)(2 \omega)^{n} B_{n}(\Lambda(\omega) .
\end{array}\right.
$$

Substituting this into (2) and using $u_{n}:=2 b_{n}, n \geq 0, v_{n}:=1-4 a_{n}$ or $a_{n}=\left(1-v_{n}\right) / 4, n \geq 1$, we obtain

$$
\begin{aligned}
& C_{n+1}=\left(\omega^{2}+1+u_{n} \omega\right) C_{n}-\omega^{2}\left(1-v_{n}\right) C_{n-1}, \\
& D_{n+1}=\left(\omega^{2}+1+u_{n} \omega\right) D_{n}-\omega^{2}\left(1-v_{n}\right) D_{n-1},
\end{aligned}
$$

or with $w:=\omega^{2}, \omega \in \mathbb{C}, v_{0}:=0$

$$
\left\{\begin{array}{rlrl}
C_{n+1}-C_{n} & =w\left(C_{n}-C_{n-1}\right)+u_{n} \omega C_{n}+v_{n} w C_{n-1}, & & n \geq 1, \\
D_{n+1}-D_{n} & =w\left(D_{n}-D_{n-1}\right)+u_{n} \omega D_{n}+v_{n} w D_{n-1}, & & n \geq 0, \\
C_{0} & =D_{-1}:=0, C_{1}=D_{0}:=1-w .
\end{array}\right.
$$

We now want to determine explicitly the two linearly independent solutions $C_{n}, D_{n}, n \geq 1$, of the difference equation (15).

Throughout this paper we will use the following fundamental definitions. Let $j, n, r, k+1 \in \mathbb{N}_{0}$ and $\omega \in \mathbb{C}$. Then we define

$$
\left\{\begin{array}{l}
c_{k, j}(\omega):=(1-w)^{-1}\left(\omega u_{j}\left(1-w^{j-k}\right)+w v_{j}\left(1-w^{j-k-1}\right)\right) \text { for } j>k \geq-1, \\
\text { with } u_{j}:=2 b_{j}, j \geq 0, v_{j}:=1-4 a_{j}, j \geq 1, v_{0}:=0, w:=\omega^{2}, \\
\text { and } c_{k, j}( \pm 1):= \pm(j-k) u_{j}+(j-k-1) v_{j},
\end{array}\right.
$$

$$
\left\{\begin{array}{l}
S_{k, 0}^{(n)}(\omega):=1-w^{n-k} \quad \text { for } n>k \geq-1 \text { and } \\
S_{k, r}^{(n)}(\omega):=\sum_{j=k+1}^{n-r} c_{k, j}(\omega) S_{j, r-1}^{(n)}(\omega) \text { for } r \geq 1, k \geq-1, n>k+r
\end{array}\right.
$$

or explicitly

$$
\begin{aligned}
S_{k, r}^{(n)}(\omega) & =\sum_{j_{1}=k+1}^{n-r} c_{k, j_{1}} \sum_{j_{2}=j_{1}+1}^{n-r+1} c_{j_{1}, j_{2}} \sum_{j_{3}=j_{2}+1}^{n-r+2} c_{j_{2}, j_{3}} \cdots \sum_{j_{r}=j_{r-1}+1}^{n-1} c_{j_{r-1}, j_{r}}\left(1-w^{n-j_{r}}\right) \\
& =\sum_{k<j_{1}<j_{2}<\cdots<j_{r}<n} c_{k, j_{1}} c_{j_{1}, j_{2}} \cdots c_{j_{r-1}, j_{r}}\left(1-w^{n-j_{r}}\right),
\end{aligned}
$$


with $j_{0}=k$, if $r=1$. Furthermore, we define

$$
S_{k}^{(n)}(\omega):=\sum_{r=0}^{n-k-1} S_{k, r}^{(n)}(\omega) \text { for } n>k \geq-1
$$

or explicitly

(19) $S_{k}^{(n)}(\omega)=1-w^{n-k}+\sum_{r=1}^{n-k-1} \sum_{k<j_{1}<j_{2}<\cdots<j_{r}<n} c_{k, j_{1}} c_{j_{1}, j_{2}} \cdots c_{j_{r-1}, j_{r}}\left(1-w^{n-j_{r}}\right)$.

Using these notations we will now prove

Lemma 1. For all $n \geq 1 C_{n}(\omega)=S_{0}^{(n)}(\omega)$ and for all $n \geq 0 \quad D_{n}(\omega)=S_{-1}^{(n)}(\omega)$ hold.

Proof. Put $t_{j}:=\omega u_{j} C_{j}+w v_{j} C_{j-1}$ for $j \geq 1$. Then the first equation of (15) becomes $C_{j+1}-C_{j}=w\left(C_{j}-C_{j-1}\right)+t_{j}, j \geq 1$. Summing from $j=1$ to $j=n-1$ and using $C_{0}=0$ yields $C_{n}=w C_{n-1}+C_{1}+\sum_{j=1}^{n-1} t_{j}, n \geq 2$. Substituting this expression into itself for $n-1, n-2, \ldots$ yields after $k \leq n-1$ steps $C_{n}=w^{k} C_{n-k}+1-w^{k}+\sum_{\nu=1}^{k} w^{\nu-1} \sum_{j=1}^{n-\nu} t_{j}$. With $C_{1}=1-w$ we obtain for $k=n-1$

$$
C_{n}=1-w^{n}+\sum_{\nu=1}^{n-1} w^{\nu-1} \sum_{j=1}^{n-\nu} t_{j}=1-w^{n}+\sum_{j=1}^{n-1} t_{j} \sum_{\nu=1}^{n-j} w^{\nu-1}
$$

or with (17)

(20)

$$
\left\{\begin{aligned}
C_{n} & =1-w^{n}+\sum_{j=1}^{n-1} t_{j}\left(1-w^{n-j}\right)(1-w)^{-1} \\
& =S_{0,0}^{(n)}+\sum_{j=1}^{n-1} t_{j} S_{j, 0}^{(n)}(1-w)^{-1}, \quad n \geq 2 .
\end{aligned}\right.
$$

For $n=2, t_{1}(1-w)^{-1}=c_{0,1}$ holds and therefore $C_{2}=S_{0,0}^{(2)}+S_{0,1}^{(2)}=S_{0}^{(2)}$ follows with (18). Assume now that for some $r \geq 1$ and all $n>r$,

$$
C_{n}=\sum_{\nu=0}^{r-1} S_{0, \nu}^{(n)}+\sum_{j=1}^{n-r} t_{j} S_{j, r-1}^{(n)}(1-w)^{-1}
$$

is already proved. We now consider the second sum only. Here we substitute 
(20) for $C_{j-1}$ and $C_{j}$ in $t_{j}$ and use (16), (17), (18). Then

$$
\begin{aligned}
& \sum_{j=1}^{n-r} t_{j} S_{j, r-1}^{(n)}(1-w)^{-1}=\sum_{j=1}^{n-r}\left(\omega u_{j} C_{j}+w v_{j} C_{j-1}\right) S_{j, r-1}^{(n)}(1-w)^{-1} \\
& =\sum_{j=1}^{n-r}\left(\omega u_{j}\left(1-w^{j}+\sum_{k=1}^{j-1} t_{k}\left(1-w^{j-k}\right)(1-w)^{-1}\right)\right. \\
& \left.\quad+w v_{j}\left(1-w^{j-1}+\sum_{k=1}^{j-2} t_{k}\left(1-w^{j-k-1}\right)(1-w)^{-1}\right)\right) S_{j, r-1}^{(n)}(1-w)^{-1} \\
& \left.=\sum_{j=1}^{n-r} c_{0, j} S_{j, r-1}^{(n)}+\sum_{j=2}^{n-r} \sum_{k=1}^{j-1} t_{k} c_{k, j} S_{j, r-1}^{(n)}(1-w)^{-1}\right) \\
& =S_{0, r}^{(n)}+\sum_{k=1}^{n-r-1} t_{k} \sum_{j=k+1}^{n-r} c_{k, j} S_{j, r-1}^{(n)}(1-w)^{-1} \\
& =S_{0, r}^{(n)}+\sum_{k=1}^{n-r-1} t_{k} S_{k, r}^{(n)}(1-w)^{-1} .
\end{aligned}
$$

Hence we have proved

$$
C_{n}=\sum_{\nu=0}^{r} S_{0, \nu}^{(n)}+\sum_{j=1}^{n-r-1} t_{j} S_{j, r}^{(n)}(1-w)^{-1} \text { for } n>r+1 .
$$

For $r=n-2$ this yields $C_{n}=\sum_{\nu=0}^{n-2} S_{0, \nu}^{(n)}+t_{1} S_{1, n-2}^{(n)}(1-w)^{-1}$. From (16) and (17) $t_{1}(1-w)^{-1}=c_{0,1}$ and $t_{1} S_{1, n-2}^{(n)}(1-w)^{-1}=c_{0,1} S_{1, n-2}^{(n)}=S_{0, n-1}^{(n)}$ follow. Hence using (18) we have proved $C_{n}=\sum_{\nu=0}^{n-1} S_{0, \nu}^{(n)}=S_{0}^{(n)}$ for $n \geq 1$. Using $D_{-1}=0$ instead of $C_{0}=0$, reasoning analogous to that above proves $D_{n}=\sum_{\nu=0}^{n} S_{-1, \nu}^{(n)}=S_{-1}^{(n)}$ for $n \geq 0$.

Our next goal is to study the asymptotic behaviour of $C_{n}, D_{n}$, as $n \rightarrow \infty$, under the assumption (3) or (4). This requires further notations. For $k+2 \in \mathbb{N}$ we define

$$
\begin{gathered}
S_{k, 0}(\omega):=1, \quad \text { and for } r \geq 1, \\
S_{k, r}(\omega):=\sum_{j=k+1}^{\infty} c_{k, j}(\omega) S_{j, r-1}(\omega),
\end{gathered}
$$

and

$$
S_{k}(\omega):=\sum_{r=0}^{\infty} S_{k, r}(\omega)
$$

or explicitly

$$
\begin{aligned}
S_{k, r}(\omega) & =\sum_{j_{1}=k+1}^{\infty} c_{k, j_{1}} \sum_{j_{2}=j_{1}+1}^{\infty} c_{j_{1}, j_{2}} \sum_{j_{3}=j_{2}+1}^{\infty} c_{j_{2}, j_{3}} \ldots \sum_{j_{r}=j_{r-1}+1}^{\infty} c_{j_{r-1}, j_{r}} \\
& =\sum_{k<j_{1}<j_{2}<\cdots<j_{r}<\infty} c_{k, j_{1}} c_{j_{1}, j_{2}} c_{j_{2}, j_{3}} \cdots c_{j_{r-1}, j_{r}},
\end{aligned}
$$


and

$$
S_{k}(\omega)=1+\sum_{r=1}^{\infty} \sum_{k<j_{1}<j_{2}<\cdots<j_{r}<\infty} c_{k, j_{1}} c_{j_{1}, j_{2}} c_{j_{2}, j_{3}} \cdots c_{j_{r-1}, j_{r}} .
$$

Next, using the maximum principle, we observe that in case $R>1$,

$$
\left|c_{k, j}(\omega)\right| \leq(R-1)^{-1}\left(\left|u_{j}\right| R^{1 / 2}\left(1+R^{j-k}\right)+\left|v_{j}\right|\left(R+R^{j-k}\right)\right)
$$

holds for $|\omega| \leq R^{1 / 2}, j>k \geq-1$, and in case $R=1$,

$$
\left|c_{k, j}(\omega)\right| \leq 2|1-w|^{-1}\left(\left|u_{j}\right|+\left|v_{j}\right|\right)
$$

holds for $|\omega| \leq 1, \omega \neq \pm 1, j>k \geq-1$. We assume that (3) holds and define for $k+2 \in \mathbb{N}$,

$$
\rho_{k}(R):=\sum_{j=k+1}^{\infty}\left(\left|u_{j}\right| R^{1 / 2}\left(1+R^{j-k}\right)+\left|v_{j}\right|\left(R+R^{j-k}\right)\right),
$$

and observe that $\rho_{k}(R) \searrow 0$ for $k \nearrow \infty$. In particular

$$
\rho_{k}(1)=2 \sum_{j=k+1}^{\infty}\left(\left|u_{j}\right|+\left|v_{j}\right|\right)
$$

Lemma 2. Assume that (3) holds with $R=1$. Then the following statements are true.

(a) For $|\omega| \leq 1, \omega \neq \pm 1, r \geq 1, k \geq-1$, and all $n>k+r$,

$$
\left|S_{k, r}^{(n)}(\omega)\right| \leq 2|1-w|^{-r} \rho_{k}(1) \rho_{k+1}(1) \cdots \rho_{k+r-1}(1) \text { holds. }
$$

(b) For each $k \geq-1, r \geq 1$, the $r$-fold series $S_{k, r}$ converges absolutely and uniformly on compact subsets of $|\omega| \leq 1, \omega \neq \pm 1$ and satisfies

$$
\left|S_{k, r}(\omega)\right| \leq|1-w|^{-r} \rho_{k}(1) \rho_{k+1}(1) \cdots \rho_{k+r-1}(1) \quad \text { for }|\omega| \leq 1, \omega \neq \pm 1 .
$$

Furthermore for each $k \geq-1, S_{k}(\omega)$ converges absolutely and uniformly on compact subsets of $|\omega| \leq 1, \omega \neq \pm 1$. Hence, for all $k \geq-1, r \geq 0, S_{k, r}$ and $S_{k}$ are holomorphic for $|\omega|<1$ and are continuous and satisfy (21), (22) for $|\omega| \leq 1, \omega \neq \pm 1$.

(c) For each $k \geq-1, r \geq 0$, and $0<t<1$,

$$
\lim _{n \rightarrow \infty} S_{k, r}^{(n)}=S_{k, r} \quad \text { and } \quad \lim _{n \rightarrow \infty} S_{k}^{(n)}=S_{k}
$$

hold uniformly for $|\omega| \leq t$.

Proof. (a) Using (24), (25), and $\left|S_{k, 0}^{(n)}\right| \leq 2$ one obtains (26) by induction on $r$ from the recursive definition of $S_{k, r}^{(n)}$ in (17).

(b) Using again (24) and (25); the convergence of $S_{k, r}$ and inequality (27) follow from $(21)$ by induction on $r$. Finally, (22), (27) and $\rho_{j}(1) \searrow 0$ for $j \nearrow \infty$ imply the convergence of $S_{k}$.

(c) Let $0<t<1$ be fixed. Then for each $k \geq-1, \lim _{n \rightarrow \infty} S_{k, 0}^{(n)}=S_{k, 0}$ holds uniformly for $|\omega| \leq t$. Let $r \geq 1$ and choose $n_{0}>k$. Then (17), (21) 
yield for $n>r+n_{0}$,

$$
\begin{aligned}
\left|S_{k, r}-S_{k, r}^{(n)}\right|= & \left|\sum_{j=k+1}^{\infty} c_{k, j} S_{j, r-1}-\sum_{j=k+1}^{n-r} c_{k, j} S_{j, r-1}^{(n)}\right| \\
\leq & \sum_{j=k+1}^{n_{0}}\left|c_{k, j}\right|\left|S_{j, r-1}-S_{j, r-1}^{(n)}\right|+\sum_{j=n_{0}+1}^{\infty}\left|c_{k, j}\right|\left|S_{j, r-1}\right| \\
& +\sum_{j=n_{0}+1}^{n-r}\left|c_{k, j}\right|\left|S_{j, r-1}^{(n)}\right| .
\end{aligned}
$$

Because of (24), (25), and (27) (applied to $S_{j, r-1}, j \geq n_{0}+1$ ) here the second sum is $\leq|1-w|^{-r} \rho_{n_{0}}(1) \rho_{n_{0}+1}(1) \cdots \rho_{n_{0}+r-1}(1)$ and because of (26) (applied to $\left.S_{j, r-1}^{(n)}, j \geq n_{0}+1\right)$ the last sum is $\leq 2|1-w|^{-r} \rho_{n_{0}}(1) \rho_{n_{0}+1}(1) \cdots \rho_{n_{0}+r-1}(1)$ for $|\omega| \leq t$. For large $n_{0}$ these upper bounds become small uniformly for $|\omega| \leq t$. We now assume that $\lim _{n \rightarrow \infty} S_{j, r-1}^{(n)}=S_{j, r-1}$ already holds uniformly on $|\omega| \leq t$ for each fixed $j \geq-1$. Then for fixed $n_{0}$ also the first sum in the above estimate becomes small for large $n$. Hence the first limit relation in (c) is proved. In order to prove the second relation we again choose a fixed $n_{0}$. Then (18), (22) yield for $n>n_{0}+k+2$,

$$
\begin{aligned}
\left|S_{k}-S_{k}^{(n)}\right| & =\left|\sum_{r=0}^{\infty} S_{k, r}-\sum_{r=0}^{n-k-1} S_{k, r}^{(n)}\right| \\
& \leq\left|\sum_{r=0}^{n_{0}}\left(S_{k, r}-S_{k, r}^{(n)}\right)\right|+\sum_{r=n_{0}+1}^{\infty}\left|S_{k, r}\right|+\sum_{r=n_{0}+1}^{n-k-1}\left|S_{k, r}^{(n)}\right| .
\end{aligned}
$$

Using again (26), (27) here the last two sums together are estimated by

$$
3 \sum_{r=n_{0}+1}^{\infty}|1-w|^{-r} \rho_{k}(1) \rho_{k+1}(1) \cdots \rho_{k+r-1}(1),
$$

which converges and for large $n_{0}$ becomes small uniformly for $|\omega| \leq t$, since $\rho_{j}(1) \searrow 0$ as $j \nearrow \infty$. Finally, for fixed $n_{0}$ the first sum becomes small for large $n$.

For $k=0,-1$ we immediately obtain from Lemmas 1 and 2

Corollary 1. Assume that (3) holds with $R=1$ and define $C(\omega):=S_{0}(\omega)$ and $D(\omega):=S_{-1}(\omega)$ for $|\omega| \leq 1, \omega \neq \pm 1$. Then the following statements are true.

(a) For every fixed $0<t<1, \lim _{n \rightarrow \infty} C_{n}(\omega)=C(\omega)$ and $\lim _{n \rightarrow \infty} D_{n}(\omega)=$ $D(\omega)$ hold uniformly for $|\omega| \leq t$.

(b) The functions $C(\omega), D(\omega)$ are holomorphic for $|\omega|<1$ and continuous for $|\omega| \leq 1, \omega \neq \pm 1$ and satisfy $C \not \equiv 0, D \not \equiv 0$, since $C(0)=D(0)=1$.

We now assume that condition (4) is satisfied. Then we obtain

$$
\left|c_{k, j}(\omega)\right| \leq(j-k)\left|u_{j}\right|+(j-k-1)\left|v_{j}\right| \text { for }|\omega| \leq 1, j>k \geq-1,
$$

and we define for $k \geq-1$,

$$
\sigma_{k}:=\sum_{j=k+1}^{\infty}\left((j-k)\left|u_{j}\right|+(j-k-1)\left|v_{j}\right|\right)
$$


observing that $\sigma_{k} \searrow 0$ for $k \nearrow \infty$. Then the results of Lemma 2 are complemented in

Lemma 3. Assume that (4) holds. Then the following statements are true.

(a) For each $k \geq-1, r>0, S_{k, r}$ in (21) converges absolutely and uniformly for $|\omega| \leq 1$ and satisfies

$$
\left|S_{k, r}(\omega)\right| \leq \sigma_{k} \sigma_{k+1} \cdots \sigma_{k+r-1} \quad \text { for }|\omega| \leq 1 \text {. }
$$

(b) For each $k \geq-1, S_{k}$ in (22) converges absolutely and uniformly for $|\omega| \leq 1$. Hence, for all $k \geq-1, r \geq 0, S_{k, r}$ and $S_{k}$ are continuous and satisfy (21), (22) for $|\omega| \leq 1$.

(c) For each $k \geq-1, S_{k}^{(n)}$ in (18) satisfies $S_{k}^{(n)}( \pm 1)=0$ and

$$
\lim _{n \rightarrow \infty}\left(\lim _{\omega \rightarrow \pm 1} S_{k}^{(n)}(\omega) /(1-w)\right) / n=S_{k}( \pm 1) .
$$

Proof. (a) Using (28), and (29); the convergence of $S_{k, r}$ and inequality (30) for $|\omega| \leq 1$ follow by induction on $r$ from the recursive definition (21).

(b) Then (22), (30), and $\sigma_{k} \searrow 0$ for $k \nearrow \infty$, imply the convergence of $S_{k}$, and (21), (22) also hold for $\omega= \pm 1$.

(c) We define for $r \geq 0, n>k+r, k \geq-1$,

$L_{k, r}^{(n)}( \pm 1):=\lim _{\omega \rightarrow \pm 1} S_{k, r}^{(n)}(\omega) /(1-w)$ and for $n>k \geq-1, L_{k}^{(n)}( \pm 1):=$ $\lim _{\omega \rightarrow \pm 1} S_{k}^{(n)}(\omega) /(1-w)$. Then $(17)$ yields $L_{k, 0}^{(n)}( \pm 1)=n-k$, and recursively for $r>0$,

$$
L_{k, r}^{(n)}( \pm 1)=\sum_{j=k+1}^{n-r} c_{k, j}( \pm 1) L_{j, r-1}^{(n)}( \pm 1)
$$

and (18) yields

$$
L_{k}^{(n)}( \pm 1)=\sum_{r=0}^{n-k-1} L_{k, r}^{(n)}( \pm 1) \text { for } n>k \geq-1 .
$$

Using (28), (29), $L_{k, 0}^{(n)}( \pm 1) / n=1-k / n \leq 2$ and the recursive definition (31), yields for $r>0, n>k+r, k \geq-1$,

$$
\left|L_{k, r}^{(n)}( \pm 1) / n\right| \leq 2 \sigma_{k} \sigma_{k+1} \cdots \sigma_{k+r-1} .
$$

Finally, using (21), (22), (30) for $\omega= \pm 1$, a repetition of the proof-idea of Lemma 2(c) with (26), and (27) replaced by (32), and (30) respectively, yields $\lim _{n \rightarrow \infty} L_{k, r}^{(n)}( \pm 1) / n=S_{k, r}( \pm 1)$ and $\lim _{n \rightarrow \infty} L_{k}^{(n)}( \pm 1) / n=S_{k}( \pm 1)$.

Looking again at (16) and (17) we observe that

$$
c_{k, j}\left(\omega^{-1}\right)=w^{k-j} c_{k, j}(\omega) \text { holds for } \omega \in \mathbb{C}, \omega \neq 0 .
$$

Consequently

$$
S_{k}^{(n)}\left(\omega^{-1}\right)=-w^{k-n} S_{k}^{(n)}(\omega) \text { holds for } \omega \in \mathbb{C}, \omega \neq 0,
$$

and $n>k \geq-1$. In particular $k=0,-1$, and Lemma 1 yield $C_{n}\left(\omega^{-1}\right)=$ $-w^{-n} C_{n}(\omega), D_{n}\left(\omega^{-1}\right)=-w^{-n-1} D_{n}(\omega), \omega \neq 0$. For fixed $k \geq-1$ we now 
want to investigate the asymptotic behaviour of $S_{k}^{(n)}$ for $|\omega|=1$ as $n \rightarrow \infty$. Using (19) we write for $n>k \geq-1$,

$$
S_{k}^{(n)}(\omega)=X_{k}^{(n)}(\omega)-w^{n} Y_{k}^{(n)}(\omega)
$$

where we have defined for $n>k \geq-1, \omega \in \mathbb{C}$,

$$
X_{k}^{(n)}(\omega):=1+\sum_{r=1}^{n-k-1} \sum_{k<j_{1}<j_{2}<\cdots<j_{r}<n} c_{k, j_{1}} c_{j_{1}, j_{2}} \cdots c_{j_{r-1}, j_{r}},
$$

and

$$
Y_{k}^{(n)}(\omega):=w^{-k}+\sum_{r=1}^{n-k-1} \sum_{k<j_{1}<j_{2}<\cdots<j_{r}<n} c_{k, j_{1}} c_{j_{1}, j_{2}} \cdots c_{j_{r-1}, j_{r}} w^{-j_{r}},
$$

where $j_{0}=k$, if $r=1$. Then we obtain immediately from (33)

$$
Y_{k}^{(n)}(\omega)=w^{-k} X_{k}^{(n)}\left(\omega^{-1}\right) \text { for all } \omega \in \mathbb{C}, \omega \neq 0 .
$$

Substituting this into (34) yields

$$
S_{k}^{(n)}(\omega)=X_{k}^{(n)}(\omega)-w^{n-k} X_{k}^{(n)}\left(\omega^{-1}\right) \text { for } \omega \in C, \omega \neq 0 .
$$

Lemma 4. Assume that (3) holds with $R=1$. Then the following statements are true.

(a) For every $k \geq-1, \lim _{n \rightarrow \infty} X_{k}^{(n)}(\omega)=S_{k}(\omega)$ holds uniformly on compact subsets of $|\omega| \leq 1, \omega \neq \pm 1$. (This holds uniformly on $|\omega| \leq 1$ if (4) is satisfied.)

(b) For fixed $k \geq-1$ and $n \rightarrow \infty$,

$$
S_{k}^{(n)}(\omega)=S_{k}(\omega)-w^{n-k} S_{k}(\bar{\omega})+o(1)
$$

holds uniformly on compact subsets of $|\omega|=1, \omega \neq \pm 1$. (This holds uniformly on $|\omega|=1$ if (4) is satisfied.)

(c) For fixed $k \geq-1$ and every fixed $|\omega|=1, \omega \neq \pm 1, \lim _{n \rightarrow \infty} S_{k}^{(n)}(\omega)$ exists, and equals $S_{k}(\omega)$, iff $S_{k}(\bar{\omega})=0$.

Proof. We observe that in analogy with (17), and (18), $X_{k}^{(n)}$ in (35) also can be defined as follows:

$X_{k}^{(n)}=\sum_{r=0}^{n-k-1} X_{k, r}^{(n)}, n>k \geq-1$, where $X_{k, 0}^{(n)}:=1$, and for $r \geq 1$, $X_{K, r}^{(n)}:=\sum_{j=k+1}^{n-r} c_{k, j} X_{j, r-1}^{(n)}$. Then instead of $S_{k, r}^{(n)}$ also $X_{k, r}^{(n)}$ satisfies inequality (26), without the factor 2 since $X_{k, 0}^{(n)}=1$. If (4) holds, then instead of $S_{k, r}$ also $X_{k, r}^{(n)}$ satisfies inequality (30). A repetition of the proof of Lemma 2(c) shows that for each $k \geq-1, r \geq 0 \lim _{n \rightarrow \infty} X_{k, r}^{(n)}=S_{k, r}$ and $\lim _{n \rightarrow \infty} X_{k}^{(n)}=S_{k}$ hold uniformly on compact subsets of $|\omega| \leq 1, \omega \neq \pm 1$. This holds uniformly on $|\omega| \leq 1$ if (4) is satisfied and (30) is used instead of (26), and (27).

(b) follows from (a) by substituting $X_{k}^{(n)}=S_{k}+o(1)$ into (36). (c) follows from (37).

Next we observe that (15) implies

$$
C_{n+1} D_{n}-D_{n+1} C_{n}=w\left(1-v_{n}\right)\left(C_{n} D_{n-1}-D_{n} C_{n-1}\right)
$$


and hence we obtain the Wronskian-type formula

$$
C_{n+1} D_{n}-D_{n+1} C_{n}=w^{n}(1-w)^{2} \prod_{j=1}^{n}\left(1-v_{j}\right) \text { for } n \geq 1,
$$

where $1-v_{j}=4 a_{j} \neq 0$ for $j \geq 1$. Since $\sum_{j=1}^{\infty}\left|v_{j}\right|<\infty, \lim _{n \rightarrow \infty} \prod_{j=1}^{n}\left(1-v_{j}\right)$ exists $\neq 0$.

Applying Lemmas 3 and 4 for $k=0,-1$ and using (38), we obtain the following result on the asymptotic behaviour of $C_{n}(\omega)$, and $D_{n}(\omega)$ for $|\omega|=1$ as $n \rightarrow \infty$.

Corollary 2. Assume that (3) holds with $R=1$. Then the following statements are true.

(a) As $n \rightarrow \infty$,

$$
C_{n}(\omega)=C(\omega)-w^{n} C(\bar{\omega})+o(1), \quad D_{n}(\omega)=D(\omega)-w^{n+1} D(\bar{\omega})+o(1)
$$

hold uniformly on compact subsets of $|\omega|=1, \omega \neq \pm 1$. If (4) is satisfied, then $C(\omega), D(\omega)$ are continuous for $|\omega| \leq 1$ and (39) holds uniformly for $|\omega|=1$.

(b) For all $|\omega|=1, \omega \neq \pm 1$,

$$
\omega^{-1} C\left(\omega^{-1}\right) D(\omega)-\omega C(\omega) D\left(\omega^{-1}\right)=\left(\omega^{-1}-\omega\right) \prod_{j=1}^{\infty}\left(1-v_{j}\right) \text { holds. }
$$

(c) For a fixed $|\omega|=1, \omega \neq \pm 1 \lim _{n \rightarrow \infty} C_{n}(\omega)$ exists, and equals $C(\omega) \neq$ 0 , iff $C(\bar{\omega})=0$. $\lim _{n \rightarrow \infty} D_{n}(\omega)$ exists, and equals $D(\omega) \neq 0$, iff $D(\bar{\omega})=0$. At least one sequence $C_{n}(\omega)$ or $D_{n}(\omega)$ diverges as $n \rightarrow \infty$.

(d) If all $a_{n}, b_{n}$ in (2) are real, then $C(\omega)=\overline{C(\bar{\omega})} \neq 0$, and $D(\omega)=\overline{D(\bar{\omega})} \neq$ 0 hold for all $|\omega|=1, \omega \neq \pm 1$ and both sequences $C_{n}(\omega), D_{n}(\omega)$ diverge for these $\omega$ as $n \rightarrow \infty$.

(e) If (4) is satisfied, then

$$
\begin{aligned}
& \lim _{n \rightarrow \infty}\left(\lim _{\omega \rightarrow \pm 1} C_{n}(\omega) /(1-w)\right) / n=C( \pm 1), \quad \text { and } \\
& \lim _{n \rightarrow \infty}\left(\lim _{\omega \rightarrow \pm 1} D_{n}(\omega) /(1-w)\right) / n=D( \pm 1) \quad \text { hold. }
\end{aligned}
$$

Proof. (b) Substituting (39) into (38) and then dividing by $w^{n}(1-w)$ yields (40).

(c) This follows from Lemma 4(c) and from the fact that because of (40); $C(\omega), C(\bar{\omega})$; or $D(\omega), D(\bar{\omega})$; or $C(\bar{\omega}), D(\bar{\omega})$ cannot both vanish.

(d) Again (40) yields $C(\omega) \neq 0$ and $D(\omega) \neq 0$ for all $|\omega|=1, \omega \neq \pm 1$.

(e) follows from Lemma 3(c).

Next, we will prove that $S_{k}(\omega)$ in (22) can be extended analytically from $|\omega|<1$ onto $|\omega|<R^{1 / 2}, R>1$, provided (3) holds for this $R>1$.

Lemma 5. Assume that (3) holds for some $R>1$. Then the following statements are true.

(a) For each $k \geq-1$, and $r>0, S_{k, r}$ in (21) converges absolutely and uniformly and satisfies

$$
\left|S_{k, r}(\omega)\right| \leq(R-1)^{-r} \rho_{k}(R) \rho_{k+1}(R) \cdots \rho_{k+r-1}(R) \quad \text { for }|\omega| \leq R^{1 / 2} .
$$


(b) For each $k \geq-1, S_{k}$ in (22) converges absolutely and uniformly for $|\omega| \leq R^{1 / 2}$. Hence, for all $k \geq-1$, and $r \geq 0, S_{k, r}$ and $S_{k}$ are holomorphic for $|\omega|<R^{1 / 2}$, continuous for $|\omega| \leq R^{1 / 2}$, and (21), and (22) hold for $|\omega| \leq R^{1 / 2}$. Proof. (a) The convergence of $S_{k, r}$ and (41) follow by induction on $r$ from the recursive definition (21) and from (23), and (25).

(b) The convergence of $S_{k}$ follows from (22), (41), and $\rho_{j}(R) \searrow 0$ as $j \nearrow$ $\infty$.

For $k=0,-1$, Lemma 5 yields

Corollary 3. Assume that (3) holds for some $R>1$. Then the functions $C(\omega)$, $D(\omega)$ are holomorphic for $|\omega|<R^{1 / 2}$, continuous for $|\omega| \leq R^{1 / 2}$, and satisfy

$$
\omega^{-1} C\left(\omega^{-1}\right) D(\omega)-\omega C(\omega) D\left(\omega^{-1}\right)=\left(\omega^{-1}-\omega\right) \prod_{j=1}^{\infty}\left(1-v_{j}\right),
$$

for $R^{-1 / 2} \leq|\omega| \leq R^{1 / 2}$.

Proof. In order to prove (42) we observe that according to Lemma 5 both sides in (42) are holomorphic for $R^{-1 / 2}<|\omega|<R^{1 / 2}$, continuous for $R^{-1 / 2} \leq|\omega| \leq$ $R^{1 / 2}$, and that equality holds for $|\omega|=1$ because of $(40)$.

We conclude this section with

Lemma 6. Assume that (3) holds. Then for every $k \geq 1$ (see (14), (22))

$$
C(\omega) D_{k}(\omega)-D(\omega) C_{k}(\omega)=S_{k}(\omega) w^{k}(1-w) \prod_{j=1}^{k}\left(1-v_{j}\right)
$$

holds for $|\omega| \leq R^{1 / 2}$ if (3) holds with $R>1$; for $|\omega| \leq 1$, and $\omega \neq \pm 1$ if (3) holds with $R=1$; and for $|\omega| \leq 1$ if (4) holds. On these sets $C$ and $D$ have no common zeros.

Proof. For fixed $k \geq 1$ we replace the sequences $a_{n}, b_{n}$ in (1) and $u_{n}, v_{n}$ in (15) by the sequences $a_{k+n}, b_{k+n}$ and $u_{k+n}, v_{k+n}, n \geq 1$, respectively. In analogy with (15) we therefore define for $n \geq 1$, and $\omega \in \mathbb{C}$,

$$
\left\{\begin{array}{l}
C_{n+1}^{*}:=\left(w+1+\omega u_{k+n}\right) C_{n}^{*}-w\left(1-v_{k+n}\right) C_{n-1}^{*}, \\
D_{n+1}^{*}:=\left(w+1+\omega u_{k+n}\right) D_{n}^{*}-w\left(1-v_{k+n}\right) D_{n-1}^{*},
\end{array}\right.
$$

where $C_{0}^{*}=D_{-1}^{*}:=0, C_{1}^{*}=D_{0}^{*}:=1-w$. Then for all $n \geq 0$, and $\omega \in \mathbb{C}$,

$$
\left\{\begin{array}{l}
(1-w) C_{k+n}=C_{k} D_{n}^{*}-w\left(1-v_{k}\right) C_{k-1} C_{n}^{*} \\
(1-w) D_{k+n}=D_{k} D_{n}^{*}-w\left(1-v_{k}\right) D_{k-1} C_{n}^{*}
\end{array}\right.
$$

holds, since by (15), and (44) both sides in (45) separately satisfy

$$
Y_{n+1}=\left(w+1+\omega u_{k+n}\right) Y_{n}-w\left(1-v_{k+n}\right) Y_{n-1} \text { for } n \geq 1 \text {, }
$$

and have the same initial values for $n=0$ and 1. Using (38) we solve (45) for $C_{n}^{*}$ and obtain

$$
C_{k+n} D_{k}-D_{k+n} C_{k}=C_{n}^{*} w^{k}(1-w) \prod_{j=1}^{k}\left(1-v_{j}\right)
$$


Because of Lemma 1, $C_{n}^{*}=S_{0}^{(n)^{*}}$ holds, where $S_{0}^{(n)^{*}}$ is obtained from $S_{0}^{(n)}$ after $u_{j}, v_{j}$ are replaced by $u_{k+j}, v_{k+j}, j \geq 0$. Now (16), and (19) show that $S_{0}^{(n)^{*}}=S_{k}^{(k+n)}$ holds for $n \geq 0$. Hence $C_{n}^{*}=S_{k}^{(k+n)}$ and also $D_{n}^{*}=S_{k-1}^{(k+n)}$ hold for $n \geq 0$ and (46) yields

$$
C_{k+n} D_{k}-D_{k+n} C_{k}=S_{k}^{(k+n)} w^{k}(1-w) \prod_{j=1}^{k}\left(1-v_{j}\right),
$$

for $n \geq 0$, and $\omega \in \mathbb{C}$. If (3) is satisfied, then by means of Lemma 2 and Corollary 1, (43) follows from (47) for $|\omega|<1$ and as $n \rightarrow \infty$. The rest follows from Lemmas 3 and 5, Corollary 3, and from $C(0)=D(0)=1, C_{k}( \pm 1)=$ $D_{k}( \pm 1)=0, \lim _{k \rightarrow \infty} S_{k}=1$.

\section{THE MAIN RESULTS CONCERNING CONTINUED FRACTIONS OF TYPE (1)}

In view of (14), (17), (18), and Lemma 1 we immediately obtain explicit formulas representing $A_{n}(\lambda)$, and $B_{n}(\lambda)$ for all $n \in \mathbb{N}$ by substituting $\omega(\lambda)$ from (5) for $\omega$ in $C_{n}(\omega), D_{n}(\omega)$. We now assume that (3) holds with $R=1$. Using (5) and the results of Corollary 1 we define

$$
A(\lambda):=2 \omega(\lambda) C(\omega(\lambda)), \quad B(\lambda):=D(\omega(\lambda)) \text { for } \lambda \in \mathbb{C}^{*} .
$$

Next let

$U:=(-1,1), L:=(-1,1)$ denote the upper and lower boundary respectively of the cut $[-1,1]$ of $\mathbb{C}^{*}$, considered as disjoint subsets of the Riemann surface $\mathbb{C}^{* *}$ of $\omega(\lambda)$.

Then for $0<\vartheta<\pi, \lambda=\cos \vartheta \in U$ implies $\omega(\lambda)=\lambda-i\left(1-\lambda^{2}\right)^{1 / 2}=e^{-i \vartheta}$, and $\lambda=\cos \vartheta \in L$ implies $\omega(\lambda)=e^{i \vartheta}$. Hence, $\omega(\lambda)$ maps the disjoint union $\mathbb{C}^{*} \cup U \cup L$ bijectively onto $|\omega| \leq 1, \omega \neq \pm 1$. Using the results of Corollary 1 again we define for $x=\cos \vartheta, 0<\vartheta<\pi$,

$$
\left\{\begin{array}{l}
A^{+}(x):=2 e^{-i \vartheta} C\left(e^{-i \vartheta}\right), \quad A^{-}(x):=2 e^{i \vartheta} C\left(e^{i \vartheta}\right), \\
B^{+}(x):=D\left(e^{-i \vartheta}\right), \quad B^{-}(x):=D\left(e^{i \vartheta}\right) .
\end{array}\right.
$$

If (4) holds, then, using Corollary 2 ,we define for $\lambda= \pm 1$,

$$
A( \pm 1):= \pm 2 C( \pm 1), \quad B( \pm 1):=D( \pm 1) .
$$

With these notations we obtain

Theorem 1. Assume that (3) holds with $R=1$. Then the following statements are true.

(a) The functions $A^{+}, B^{+}, A^{-}, B^{-}$in $(50)$ are continuous on $(-1,1)$ and for every $x=\cos \vartheta, 0<\vartheta<\pi$ they satisfy

$$
\begin{aligned}
& A^{-}(x) B^{+}(x)-A^{+}(x) B^{-}(x) \\
& \quad=4 i\left(1-x^{2}\right)^{1 / 2} \prod_{j=1}^{\infty}\left(1-v_{j}\right)=4 i \sin \vartheta \prod_{j=1}^{\infty}\left(1-v_{j}\right) \neq 0 .
\end{aligned}
$$

If, in particular, all $a_{n}, b_{n}$ in (1) are real, then $A^{-}(x)=\overline{A^{+}(x)} \neq 0$, and $B^{-}(x)=\overline{B^{+}(x)} \neq 0$ hold for all $x \in(-1,1)$. 
(b) The functions $A, B$ in (48) are holomorphic and $\not \equiv 0$ on $\mathbb{C}^{*} \cup\{\infty\}$ and can be extended continuously onto $\mathbb{C}^{*} \cup U \cup L$ (see (49)). More explicitly, $A(\lambda)$, and $B(\lambda)$ approach the continuous boundary values $A^{+}(x), B^{+}(x)$ or $A^{-}(x), B^{-}(x)$ if $\lambda \in \mathbb{C}^{*}$ approaches $x \in U$ or $x \in L$ respectively. Furthermore, $A$, and $B$ do not vanish simultaneously on $\mathbb{C}^{*} \cup U \cup L$. Finally, $f(\lambda):=\lim _{n \rightarrow \infty} A_{n}(\lambda) / B_{n}(\lambda)=A(\lambda) / B(\lambda)$ holds uniformly on compact subsets of $\mathbb{C}^{*} \backslash\left\{\lambda \in \mathbb{C}^{*}: B(\lambda)=0\right\}$.

(c) For $x=\cos \vartheta, 0<\vartheta<\pi$, the continued fraction (1) diverges. More precisely $A_{n}(x) / B_{n}(x)=M\left(e^{-i 2(n+1) \vartheta}\right)+o(1)$ holds (uniformly on compact subsets of $(-1,1)$ ) as $n \rightarrow \infty$, where in view of $(52)$

$$
M(\zeta):=\left(A^{+}(x)-\zeta A^{-}(x)\right) /\left(B^{+}(x)-\zeta B^{-}(x)\right)
$$

is a Moebius transformation. Consequently, for fixed $x \in(-1,1)$, asymptotically all $A_{n}(x) / B_{n}(x)$ lie on the image circle of the unit circle under $M(\zeta)$, which is a straight line iff $\left|B^{+}(x)\right|=\left|B^{-}(x)\right|$.

(d) If (4) holds, then in addition to (a) $A$, and $B$ can be extended continuously from $\mathbb{C}^{*} \cup U \cup L$ into \pm 1 , and $A(\lambda), B(\lambda)$ approach $A( \pm 1), B( \pm 1)$ (see (51)) as $\lambda \in \mathbb{C}^{*} \cup U \cup L$ approaches \pm 1 . Neither $A(1), B(1)$ nor $A(-1), B(-1)$ vanish simultaneously. Finally, $\lim _{n \rightarrow \infty} A_{n}( \pm 1) / B_{n}( \pm 1)=A( \pm 1) / B( \pm 1)$ holds.

Proof. (a) The continuity of $A^{+}, B^{+}, A^{-}$, and $B^{-}$follows from Corollary $1(\mathrm{~b})$. Using $\omega(x)=e^{-i \vartheta}$ for $x=\cos \vartheta \in U$ and substituting (50) into (40) yields (52) and the rest follows from Corollary $2(\mathrm{~d})$,

(b) follows by combining (5), (14), (48), (49), and (50) with Corollary 1 and Lemma 6 ,

(c) follows from Corollary 2(a) by substituting $\omega(x)=e^{-i \vartheta}$, and $x=\cos \vartheta \in$ $U$, into (14), (39), and by using (50),

(d) follows from Corollary 2(a), Lemma 6 and Corollary 2(e) together with (5), (14), (51).

Remark 1. That (1) diverges for every $x \in(-1,1)$ also can be seen from the estimate

$$
\left|\frac{A_{n+1}(x)}{B_{n+1}(x)}-\frac{A_{n}(x)}{B_{n}(x)}\right| \geq 2|1-w|^{2} K^{-2} \prod_{j=1}^{n}\left|1-v_{j}\right|,
$$

which follows with $x=\cos \vartheta, \omega=e^{ \pm i \vartheta}$ from (5), (14), (38) and, as a consequence of Lemma 1, (18), (26) with $k=-1$, from

$$
\left|D_{n}(\omega)\right| \leq K=K(\omega):=2\left(1+\sum_{r=1}^{\infty}|1-w|^{-r} \rho_{-1}(1) \rho_{0}(1) \cdots \rho_{r-2}(1)\right) .
$$

Remark 2. Let all $b_{n}, n \geq 0$, be real and all $a_{n}>0, n \geq 1$, and define $I_{j}:=\left(-b_{j}-a_{j}^{1 / 2}-a_{j+1}^{1 / 2},-b_{j}+a_{j}^{1 / 2}+a_{j+1}^{1 / 2}\right) \subset \mathbb{R}$ for $j \geq 0$ (here $a_{0}:=0$ ). Let $J_{0}$ and $J_{1}$ be the smallest closed interval with $\bigcup_{j=0}^{\infty} I_{j} \subset J_{0}$ and $\bigcup_{j=1}^{\infty} I_{j} \subset J_{1}$ respectively. Then Theorem 4 in [10] shows that all zeros of $B_{n}(\lambda), n \geq 1$, are contained in $J_{0}$ and all zeros of $A_{n}(\lambda), n \geq 2$, are contained in $J_{1}$. Hence, $A$ and $B$ in (48) are $\neq 0$ on $\mathbb{C} \backslash J_{0}$ and $f(\lambda)$ in (1) is holomorphic there.

Theorem 2. Assume that (3) holds for some $R>1$. Then the following statements are true. 
(a) The functions $A, B$ in (48) can be extended analytically from $\mathbb{C}^{*}$ across $U$ and $L$ (see (49)) onto a subregion $\left(|\omega(\lambda)|<R^{1 / 2}\right)$ of the Riemann surface $\mathbb{C}^{* *}$ of $\omega(\lambda)$ (see (5)) whose boundary on $\mathbb{C}^{* *}\left(|\omega(\lambda)|=R^{1 / 2}\right.$ ) lies above the ellipse $E(R)$ (see (6)). Onto this boundary $A$ and $B$ can be extended continuously. The focal points \pm 1 of $E(R)$ are first order algebraic branch points for $f(\lambda)=A(\lambda) / B(\lambda)$ in (1). Furthermore, $A$ and $B$ have no common zeros in their extended range of definition $\left(|\omega(\lambda)| \leq R^{1 / 2}\right)$.

(b) If $\tilde{A}(\lambda), \widetilde{B}(\lambda)$ denote the values of $A, B$ obtained after extending $A, B$ analytically from $\lambda \in \mathbb{C}^{*}$ across $U$ or $L$ into the point on $\mathbb{C}^{* *}$ lying above $\lambda$, then

$$
\tilde{A}(\lambda) B(\lambda)-A(\lambda) \widetilde{B}(\lambda)=4\left(\lambda^{2}-1\right)^{1 / 2} \prod_{j=1}^{\infty}\left(1-v_{j}\right)
$$

holds for all $\lambda \in \mathbb{C}^{*}$ which lie inside or on $E(R)$ (i.e., $R^{-1 / 2} \leq|\omega(\lambda)| \leq R^{1 / 2}$ ), where $\left(\lambda^{2}-1\right)^{1 / 2}>0$ for $\lambda>1, \lambda \in \mathbb{C}^{*}$. For $\lambda \in U$ or $\lambda \in L$, (53) reduces to (52).

(c) If (3) holds for all $R>1$, then $A$ and $B$ can be extended analytically onto the complete Riemann surface $\mathbb{C}^{* *}$ and satisfy (53) for all $\lambda \in \mathbb{C}$. Then $f$ is a meromorphic function on $\mathbb{C}^{* *}$.

Proof. (a) follows from Corollary 3 and Lemma 6 in view of (5), and (48).

(b) Corollary $3,(5)$, and (48) yield $\widetilde{A}(\lambda)=2 \omega(\lambda)^{-1} C\left(\omega(\lambda)^{-1}\right), \widetilde{B}(\lambda)=$ $D\left(\omega(\lambda)^{-1}\right)$. Then (53) follows from (42).

Remark 3. If in (1) $\lim _{n \rightarrow \infty} b_{n}=0$ and $\lim _{n \rightarrow \infty} a_{n}=0$, then, as a consequence of Worpitzky's Theorem [6, 8, 15], (1) converges and $f$ is meromorphic on $\mathbb{C} \backslash\{0\}$.

Remark 4. Assume that (3) or (4) holds. Then substitution of (14) and (48) into (43) yields

$$
A(\lambda) B_{k}(\lambda)-B(\lambda) A_{k}(\lambda)=(2 \omega(\lambda))^{k+1} S_{k}(\omega(\lambda)) \prod_{j=1}^{k} a_{j},
$$

for all $\lambda$ for which $\omega(\lambda)$ satisfies the corresponding conditions of Lemma 6.

\section{ApPlications to ORTHOgONAL POLYNOMIALS}

In view of the recurrence relations (2) the sequences $B_{n}(\lambda), A_{n+1}(\lambda), n \geq 0$, now are interpreted as two sequences of orthogonal polynomials of first and second kind respectively. Using (14), (5), and Lemma 1, one obtains explicit formulas for $B_{n}, A_{n+1}$ for all $n$, and all possible complex orthogonal polynomial sequences are obtained in this way.

Assuming that (3) holds with $R=1$ or that (4) is satisfied we now are going to apply the results of $\S 2$. In the following considerations, Corollaries 1 and 2 and especially (39), (40) in Corollary 2 play a fundamental role. Recall that (40) was obtained via (39) from (38) which is a Wronskian-type relation reflecting the linear independence of the solutions $A_{n}, B_{n}, n \geq 0$, of (2). We will formulate all results for the sequence $B_{n}, n \geq 0$, only since analogous results hold for $A_{n+1}, n \geq 0$ (see Remark 5). 
If (4) is satisfied and $D(\omega)$ is $\neq 0$ for all $|\omega| \leq 1$, then, using (50), we define

$$
\phi(x):=(2 / \pi)\left(1-x^{2}\right)^{1 / 2} \prod_{j=1}^{\infty}\left(1-v_{j}\right) / B^{+}(x) B^{-}(x),
$$

for $-1 \leq x \leq 1$, where the root is $\geq 0$. Substituting $x=\cos \vartheta, 0 \leq \vartheta \leq \pi$, into $(54)$ yields $\phi(\cos \vartheta)=(2 / \pi) \sin \vartheta \prod_{j=1}^{\infty}\left(1-v_{j}\right) / D\left(e^{i \vartheta}\right) D\left(e^{-i \vartheta}\right)$, for $0 \leq \vartheta \leq$ $\pi$. Observe that because of (30) in Lemma 3, (21), and (22), $D(\omega)=S_{-1}(\omega)$ is $\neq 0$ for $|\omega| \leq 1$ if $\sum_{r=1}^{\infty} \sigma_{-1} \sigma_{0} \cdots \sigma_{r-2}<1$, and especially, if $\sigma_{-1}<1 / 2$. If $D(\omega)$ has zeros on $|\omega|=1$, then still $1 / \phi(x)$ is continuous for $-1<x<1$. In view of (50), (51), and Theorem 1(b) we define

$$
f^{+}(x):=A^{+}(x) / B^{+}(x), \quad f^{-}(x):=A^{-}(x) / B^{-}(x) \quad \text { for }-1 \leq x \leq 1 .
$$

Then (52), and (54) imply

$$
2 \pi i \phi(x)=f^{-}(x)-f^{+}(x) \text { for }-1 \leq x \leq 1,
$$

or by virtue of $(50)$, and $(40)$

$$
\pi i \phi(\cos \vartheta)=e^{i \vartheta} C\left(e^{i \vartheta}\right) / D\left(e^{i \vartheta}\right)-e^{-i \vartheta} C\left(e^{-i \vartheta}\right) / D\left(e^{-i \vartheta}\right), \quad \text { for } 0 \leq \vartheta \leq \pi .
$$

With these notations we obtain

Theorem 3. Assume that $a_{n}, b_{n} \in \mathbb{C}, a_{n} \neq 0, n \geq 0\left(a_{0}:=1\right)$ satisfy (4) and that $D(\omega)$ is $\neq 0$ for all $|\omega| \leq 1$. Then the complex function $\phi(x)$ in (54) is continuous for $-1 \leq x \leq 1$ and $\neq 0$ for $-1<x<1 \quad(\phi( \pm 1)=0)$. For all $\lambda \in \mathbb{C}^{*}(\operatorname{see}(1))$

$$
f(\lambda)=\int_{-1}^{1} \phi(x)(\lambda-x)^{-1} d x \quad \text { holds }
$$

If $\gamma$ denotes a large circle around 0 , then

$$
\begin{gathered}
\int_{-1}^{1} B_{m}(x) B_{n}(x) \phi(x) d x=\frac{1}{2 \pi i} \int_{\gamma} B_{m}(\lambda) B_{n}(\lambda) f(\lambda) d \lambda \\
=a_{0} a_{1} \cdots a_{m} \delta_{m, n} \quad \text { holds for all } m, n \geq 0 .
\end{gathered}
$$

Proof. Using (48)-(51) and because of Theorem $1(\mathrm{a}),(\mathrm{b}), f(\lambda)=A(\lambda) / B(\lambda)$ is holomorphic for $\lambda \in \mathbb{C}^{*}$ and continuous on $\mathbb{C}^{*} \cup U \cup L \cup\{1,-1\}$. For fixed $\lambda \in \mathbb{C}^{*}$ Cauchy's integral formula yields

$$
f(\lambda)=\frac{1}{2 \pi i}\left(\int_{\gamma_{1}} f(t)(t-\lambda)^{-1} d t-\int_{\gamma_{2}} f(t)(t-\lambda)^{-1} d t\right),
$$

where $\gamma_{1}$ is a large circle around 0 with $\lambda$ in its interior and $\gamma_{2}$ is a closed path surrounding $[-1,1]$ with $\lambda$ in its exterior, both curves being simple and positively oriented. Because of $\lambda f(\lambda) \rightarrow 1$ for $\lambda \rightarrow \infty$ the integral along $\gamma_{1}$ tends to 0 when the radius of $\gamma_{1}$ tends to $\infty$. Finally, $\gamma_{2}$ is contracted onto $[-1,1]$. Then $(55)$ and Theorem $1(a),(b)$ yield

$$
\begin{aligned}
f(\lambda) & =-\frac{1}{2 \pi i}\left(\int_{1}^{-1} f^{+}(x)(x-\lambda)^{-1} d x+\int_{-1}^{1} f^{-}(x)(x-\lambda)^{-1} d x\right) \\
& =\frac{1}{2 \pi i} \int_{-1}^{1}\left(f^{-}(x)-f^{+}(x)\right)(\lambda-x)^{-1} d x
\end{aligned}
$$


and hence (57) follows from (56). Next, using the same idea of proof as in [2, p. 255], we substitute (57) for $f(\lambda)$ into the right integral in (58). Inverting here the order of integration and using Cauchy's integral formula yields the left equality in (58). Substituting $f(\lambda)=A_{m}(\lambda) / B_{m}(\lambda)+a_{1} \cdots a_{m} \lambda^{-2 m-1}+$ $O\left(\lambda^{-2 m-2}\right)$, for $\lambda \rightarrow \infty$, (see Remark 4 and (5), (14), (48)) into the right-hand integral in (58) yields the second equality for $n \leq m$, when the radius of $\gamma$ tends to $\infty$ (see also Theorem 2.3. in [1]).

If $b_{n} \in \mathbb{R}$ and $a_{n}>0$ for $n \geq 0$ and if (3) holds with $R=1$, then automatically $D(\omega)=\overline{D(\bar{\omega})}$ is $\neq 0$ for $|\omega|=1, \omega \neq \pm 1$ because of Corollary $2(d)$. In this case $(54)$ yields

$$
\phi(x)=(2 / \pi)\left(1-x^{2}\right)^{1 / 2} \prod_{j=1}^{\infty}\left(1-v_{j}\right) /\left|B^{+}(x)\right|^{2}
$$

for $-1<x<1$, or

$$
\phi(\cos \vartheta)=(2 / \pi) \sin \vartheta \prod_{j=1}^{\infty}\left(1-v_{j}\right) /\left|D\left(e^{i \vartheta}\right)\right|^{2} \quad \text { for } 0<\vartheta<\pi .
$$

Now (56) holds for $-1<x<1$. In this case we also use the fact that (see (1))

$$
f(\lambda)=\int_{-a}^{a}(\lambda-x)^{-1} d \psi(x) \text { holds for all } \lambda \in \mathbb{C} \backslash[-a, a]
$$

(see Theorem 2.4. in [1] and also Remark 2), where with suitable $a \geq 1, \psi(x)$ is a real-valued nondecreasing function on $[-a, a]$, normalized by $\psi(x)=$ $\psi(x+0)$ for all $x \in(-a, a)$. Then we obtain the following result essentially due to Nevai [7, Theorem 40, p. 143].

Theorem 4. Assume that $b_{n} \in \mathbb{R}, a_{n}>0, n \geq 0,\left(a_{0}:=1\right)$ satisfy (3) with $R=1$. Then (see (60), (59)) $\psi^{\prime}(x)=\phi(x)$ holds and $\phi(x)$ is continuous and $>0$ for $-1<x<1$. If (4) holds, then $\left(1-x^{2}\right)^{1 / 2} \phi(x)$ is bounded for $-1<x<1$ or $\sin \vartheta \phi(\cos \vartheta)$ is bounded for $0<\vartheta<\pi$.

Proof. Because of Theorem 1(a), (b) and (55), (49), $f(\lambda)$ continuously approaches $f^{+}(x)$ and $f^{-}(x)$ as $\lambda \in \mathbb{C}^{*}$ approaches $x \in U$ and $x \in L$ respectively. Here $f^{-}(x)=\overline{f^{+}(x)}$ is continuous, $\neq 0$, and satisfies (56) for $-1<x<1$, where now $\phi(x)$ has the special form (59). Hence using (56) and applying the Stieltjes inversion formula (Theorem 2.5 in [1]) to (60) yields $\psi^{\prime}(x)=\phi(x)$ for $-1<x<1$ and the properties of $\phi(x)$ follow from (59). If, finally, (4) is valid, then by Corollary $2(\mathrm{a}),(\mathrm{d}), C(\omega)$ and $D(\omega)$ are continuous for $|\omega|=1$ and $\neq 0$ for $|\omega|=1, \omega \neq \pm 1$. If $D( \pm 1) \neq 0$, then $\sin \vartheta /\left|D\left(e^{i \vartheta}\right)\right|$ and hence $\sin \vartheta \phi(\cos \vartheta)$ is bounded on $0<\vartheta<\pi$. With $x=\cos \vartheta, \omega(x)=e^{-i \vartheta}, 0<\vartheta<\pi,(40)$ yields

$$
e^{i \vartheta} C\left(e^{i \vartheta}\right) D\left(e^{-i \vartheta}\right) / \sin \vartheta-e^{-i \vartheta} C\left(e^{-i \vartheta}\right) D\left(e^{i \vartheta}\right) / \sin \vartheta=2 i \prod_{j=1}^{\infty}\left(1-v_{j}\right) .
$$

If $D(1)=0$ or $D(-1)=0$, then we assume that there exist $0<\vartheta_{n}<\pi$ with $\vartheta_{n} \rightarrow 0$ or $\vartheta_{n} \rightarrow \pi$ such that $D\left(e^{i \vartheta_{n}}\right) / \sin \vartheta_{n} \rightarrow 0$ for $n \rightarrow \infty$. Then for the conjugate $D\left(e^{-i \vartheta_{n}}\right) / \sin \vartheta_{n} \rightarrow 0$ holds and $C\left(e^{i \vartheta_{n}}\right) \rightarrow C(1)$ or $C(-1)$ as $n \rightarrow \infty$. Hence, for $\vartheta=\vartheta_{n}, n \rightarrow \infty$, the left side of $(61)$ tends to 0 , whereas 
the right side of $(61)$ is a constant $\neq 0$. Therefore $\left|D\left(e^{i \vartheta}\right) / \sin \vartheta\right| \geq$ const . $>0$ holds for $0<\vartheta<\pi$, which proves that $\sin \vartheta \phi(\cos \vartheta)$ also is bounded on $0<\vartheta<\pi$ if $D( \pm 1)=0$.

In the following result (63) and (c) essentially are due to Nevai [7, Theorem 40, p. 143].

Theorem 5. Assume that $a_{n}, b_{n} \in \mathbb{C}, a_{n} \neq 0, n \geq 0 \quad\left(a_{0}:=1\right)$ satisfy (3) with $R=1$ and define $Q_{n}(\lambda):=B_{n}(\lambda) /\left(a_{0} a_{1} \cdots a_{n}\right)^{1 / 2}$, where for each $n \geq 1$, $\left(a_{0} a_{1} \cdots a_{n}\right)^{1 / 2}=2^{-n}\left(\prod_{j=1}^{n}\left(1-v_{j}\right)\right)^{1 / 2} \neq 0$ is chosen in $\{z \in \mathbb{C}:-\pi / 2 \leq \arg z<$ $\pi / 2\}$. Then the following statements are true.

(a) For $n \rightarrow \infty$ and fixed $0<t<1$ (see (5), (48))

$$
(\omega(\lambda))^{n+1} Q_{n}(\lambda)=B(\lambda) / 2\left(\lambda^{2}-1\right)^{1 / 2}\left(\prod_{j=1}^{n}\left(1-v_{j}\right)\right)^{1 / 2}+o(1)
$$

holds uniformly for $|\omega(\lambda)| \leq t$, i.e., uniformly for $\lambda$ on and outside the ellipse $E\left(t^{-2}\right)$ in (6), where $\left(\lambda^{2}-1\right)^{1 / 2}>0$ for $\lambda>1$.

(b) For $n \rightarrow \infty$

$2 i \sin \vartheta Q_{n}(\cos \vartheta)$

$$
=\left(D\left(e^{-i \vartheta}\right) e^{i(n+1) \vartheta}-D\left(e^{i \vartheta}\right) e^{-i(n+1) \vartheta}\right) /\left(\prod_{j=1}^{n}\left(1-v_{j}\right)\right)^{1 / 2}+o(1)
$$

and, consequently,

(63)

$$
\begin{aligned}
& Q_{n}^{2}(\cos \vartheta)-Q_{n-1}(\cos \vartheta) Q_{n+1}(\cos \vartheta)=D\left(e^{-i \vartheta}\right) D\left(e^{i \vartheta}\right) / \prod_{j=1}^{n}\left(1-v_{j}\right)+o(1) \\
& =(2 / \pi) \sin \vartheta / \phi(\cos \vartheta)+o(1)
\end{aligned}
$$

hold uniformly on compact subsets of $0<\vartheta<\pi$. If (4) is valid, then (62) holds uniformly on $0 \leq \vartheta \leq \pi$.

(c) If $b_{n} \in \mathbb{R}$ and $a_{n}>0$ for all $n \geq 0$ and if $\Delta(\vartheta):=\arg D\left(e^{i \vartheta}\right)$ is chosen as continuous function on $0<\vartheta<\pi$, then (62) reduces to

$$
\sin \vartheta Q_{n}(\cos \vartheta)=\left|D\left(e^{i \vartheta}\right)\right| \sin ((n+1) \vartheta-\Delta(\vartheta)) /\left(\prod_{j=1}^{n}\left(1-v_{j}\right)\right)^{1 / 2}+o(1)
$$

uniformly on compact subsets of $0<\vartheta<\pi$.

Proof. The asymptotic formula in (a) follows from (14), Corollary 1, (5), (48). In (b) (62) follows from (14), $\omega(\cos \vartheta)=e^{-i \vartheta}$ and (39) in Corollary 2(a). In 
order to prove (63) we substitute $(62)$ in

$$
\begin{aligned}
-4 \sin ^{2} \vartheta & \prod_{j=1}^{n}\left(1-v_{j}\right)\left(Q_{n}^{2}(\cos \vartheta)-\left(a_{n+1} / a_{n}\right)^{1 / 2} Q_{n-1}(\cos \vartheta) Q_{n+1}(\cos \vartheta)\right) \\
= & \left(D\left(e^{-i \vartheta}\right) e^{i(n+1) \vartheta}-D\left(e^{i \vartheta}\right) e^{-i(n+1) \vartheta}\right)^{2} \\
& -\left(D\left(e^{-i \vartheta}\right) e^{i n \vartheta}-D\left(e^{i \vartheta}\right) e^{-i n \vartheta}\right)\left(D\left(e^{-i \vartheta}\right) e^{i(n+2) \vartheta}-D\left(e^{i \vartheta}\right) e^{-i(n+2) \vartheta}\right) \\
& +o(1)=-2 D\left(e^{-i \vartheta}\right) D\left(e^{i \vartheta}\right)+D\left(e^{-i \vartheta}\right) D\left(e^{i \vartheta}\right)\left(e^{-i 2 \vartheta}+e^{i 2 \vartheta}\right)+o(1) \\
= & -4 \sin ^{2} \vartheta D\left(e^{i \vartheta}\right) D\left(e^{-i \vartheta}\right)+o(1) .
\end{aligned}
$$

Here the roots $\left(a_{n+1} / a_{n}\right)^{1 / 2}$ were chosen such that $\lim _{n \rightarrow \infty}\left(a_{n+1} / a_{n}\right)^{1 / 2}=1$. Dividing the preceding result by $-4 \sin ^{2} \vartheta \prod_{j=1}^{n}\left(1-v_{j}\right)$ yields the first equality in (63). The second one follows from (54) for $x=\cos \vartheta$.

(c) follows from (62) since according to Corollary $2(\mathrm{~d})$ now $D\left(e^{-i \vartheta}\right)=$ $\overline{D\left(e^{i \vartheta}\right)} \neq 0$ holds for $0<\vartheta<\pi$.

Remark 5. If instead of $f_{1}(\lambda):=f(\lambda)$ and corresponding $\phi_{1}(x):=\phi(x)$ in (54) we consider the continued fraction of type (1)

$$
f_{2}(\lambda):=a_{1}^{-1}\left(\lambda+b_{0}-\frac{1}{f_{1}(\lambda)}\right)=\frac{1}{\lambda+b_{1}}-\frac{a_{2}}{\lambda+b_{2}}-\frac{a_{3}}{\lambda+b_{3}}-\cdots
$$

and corresponding $\phi_{2}(x)$ of type (54), then the polynomials $A_{n+1}, n \geq 0$, now play the same role for $f_{2}(\lambda)$ which the polynomials $B_{n}, n \geq 0$, play for $f_{1}(\lambda)$. For $n \geq 0, A_{n+1}$ is the denominator of the $n$th approximant of $f_{2}(\lambda)$. If for $-1<x<1, f_{2}^{+}(x), f_{2}^{-}(x)$ denote the continuous boundary values of $f_{2}(\lambda)$ on $U$ and $L$ respectively (see (49)), then the above definition of $f_{2}$ yields $f_{2}^{-}(x)-f_{2}^{+}(x)=a_{1}^{-1}\left(1 / f^{+}(x)-1 / f^{-}(x)\right)$ for $-1<x<1$. Then (52), (55), (56), and $4 a_{1}=1-v_{1}$ imply

$$
\phi_{2}(x)=4(2 / \pi)\left(1-x^{2}\right)^{1 / 2} \prod_{j=2}^{\infty}\left(1-v_{j}\right) / A^{+}(x) A^{-}(x),
$$

or with $x=\cos \vartheta, 0<\vartheta<\pi$ and using (50)

$$
\phi_{2}(\cos \vartheta)=(2 / \pi) \sin \vartheta \prod_{j=2}^{\infty}\left(1-v_{j}\right) / C\left(e^{-i \vartheta}\right) C\left(e^{i \vartheta}\right)
$$

and, moreover,

$$
\phi_{2}(\cos \vartheta)\left(1-v_{1}\right) C\left(e^{-i \vartheta}\right) C\left(e^{i \vartheta}\right)=\phi_{1}(\cos \vartheta) D\left(e^{-i \vartheta}\right) D\left(e^{i \vartheta}\right) .
$$

Obviously analogous results as stated in Theorems 3-5 also hold for $f_{2}, \phi_{2}$, and $A_{n+1}, n \geq 0$.

As an example we consider (1) with $b_{n}:=0, n \geq 0, v_{1}:=-1, v_{n}:=0$, $n \geq 2$. Then (14), (16), (17), (19), and Lemma 1 with $\omega(\cos \vartheta)=e^{-i \vartheta}, 0<$ $\vartheta<\pi$, yield the Chebyshev polynomials of first and second kind $B_{n}(\cos \vartheta)=$ $2^{-n}\left(e^{i n \vartheta}+e^{-i n \vartheta}\right)=2^{1-n} \cos n \vartheta, n \geq 1$,

$$
\begin{aligned}
A_{n+1}(\cos \vartheta) & =2^{-n}\left(e^{i(n+1) \vartheta}-e^{-i(n+1) \vartheta}\right)\left(e^{i \vartheta}-e^{-i \vartheta}\right)^{-1} \\
& =2^{-n}(\sin (n+1) \vartheta) / \sin \vartheta, \quad n \geq 0
\end{aligned}
$$


From (22), (50), (54), Corollary 1, and Remark 5 we obtain $D(\omega)=1-\omega^{2}$, $C(\omega)=1$ and the corresponding weight functions $\phi_{1}(x)=(1 / \pi)\left(1-x^{2}\right)^{-1 / 2}$, $\phi_{2}(x)=(2 / \pi)\left(1-x^{2}\right)^{1 / 2},-1<x<1$.

Remark 6. Assume that (3) holds with $R=1$. For $|\omega| \leq 1, \omega \neq \pm 1$, and $|z|<$ 1 let $G_{k}(z):=\sum_{n=k+1}^{\infty} S_{k}^{(n)} z^{n}$ be the generating function of the sequence $S_{k}^{(n)}$, $n>k$. Using the explicit formula (19) for $S_{k}^{(n)}$ and interchanging successively the summation with respect to $n$ with all summations in (19) and summing geometric series one obtains the following explicit series representation (64)

$$
G_{k}(z)=\frac{z^{k+1}(1-w)}{(1-z)(1-z w)}\left(1+\sum_{r=1}^{\infty} \sum_{k<j_{1}<\cdots<j_{r}<\infty} c_{k, j_{1}} c_{j_{1}, j_{2}} \cdots c_{j_{r-1}, j_{r}} z^{j_{r}-k}\right),
$$

which converges absolutely for $|\omega| \leq 1, \omega \neq \pm 1,|z| \leq 1$. Moreover,

$$
\lim _{z \rightarrow 1}(1-z) G_{k}(z)=S_{k}
$$

holds (see (22)). Furthermore, it is easy to see that the series on the right side of (64) converges absolutely if $\sum_{j=k+1}^{\infty}\left|c_{k, j}(\omega) z^{j}\right|<\infty$, especially if $|z|<1$, $|\omega| \leq 1$, and the sequences $u_{j}, v_{j}$ in $c_{k, j}($ see (16)) are bounded for $j>k \geq$ -1 .

Remark 7. From Theorems 3-5 we immediately obtain more general versions and supplements with shorter proofs of the following results which are proved for real $a_{n}, b_{n}, n \geq 0$, in P. G. Nevai [7]: Theorem 40 (p. 143), Theorem 42 (p. 145), Theorem 12 (p. 12), Theorem 27 (p. 136), Theorem 29 (p. 137), Theorem 34 (p. 140) and Corollaries 35 and 36 (p. 141). In particular, Theorem 2.7 (p. 13) in [1] follows directly from (54) and Theorem 5 (c).

\section{THE MAIN RESUlTS CONCERNING CONTINUED FRACTIONS OF TYPE (7)}

In order to obtain explicit results on the continued fraction (7) which are analogous to those given for $(1)$ in $\S 3$, we observe that all identities and estimates in $\S 2$ remain valid if for $a_{n}, b_{n}$, and $\lambda$ we substitute functions $a_{n}(z), b_{n}(z)$ and $\lambda(z), n \geq 0$, having the properties described below (7) in $\S 1$. We generally refer to the notations following (7), especially to (8)-(11) and also to

Remark 8. The point $z_{0} \in S=\lambda^{-1}([-1,1])$ is a branch point of $\hat{\omega}(z)$ (extended onto $\left.G^{* *}\right)$ iff $\lambda\left(z_{0}\right)= \pm 1$ of odd order.

Assuming that (9) or (10) is valid, again the estimates (26), (27), (30), (41) play a fundamental role. They now depend on $z$ and the series in (25) and (29) converge uniformly with respect to $z$ in compact sets. After having substituted the functions $a_{n}(z), b_{n}(z)$ for the coefficients $a_{n}, b_{n}, n \geq 0$, in $C(\omega)=S_{0}(\omega)$ and $D(\omega)=S_{-1}(\omega)$ (see Corollary 1 and (22)), we will use the following more detailed notation

$$
C(z, \omega):=C(\omega), \quad D(z, \omega):=D(\omega) .
$$

Then, using $\hat{\omega}(z)$ from (11) with $|\hat{\omega}(z)|<1$ on each component of $G^{*}$, we define (see also (48), (50), (51))

$$
\widehat{A}(z):=2 \hat{\omega}(z) C(z, \hat{\omega}(z)), \quad \widehat{B}(z):=D(z, \hat{\omega}(z)) \text { for } z \in G^{*} .
$$


For each $z \in S$ with $\lambda(z) \neq \pm 1$ we can write

$$
\hat{\omega}(z)=e^{i \vartheta(z)} \quad \text { with real } \vartheta(z) \neq k \pi, k \in \mathbb{Z} .
$$

Then (40) in Corollary 2 yields

$$
\begin{aligned}
e^{i \vartheta(z)} & C\left(z, e^{i \vartheta(z)}\right) D\left(z, e^{-i \vartheta(z)}\right)-e^{-i \vartheta(z)} C\left(z, e^{-i \vartheta(z)}\right) D\left(z, e^{i \vartheta(z)}\right) \\
& =2 \sin \vartheta(z) \prod_{j=1}^{\infty}\left(1-v_{j}(z)\right) \neq 0 .
\end{aligned}
$$

Next we want to define regions $G_{0} \neq \varnothing, G_{0} \subset G^{*}$ which touch the cut $S$ only "from one side". By this we mean that $G_{0}$ satisfies (see (49))

$$
\lambda\left(G_{0} \cup\left(\bar{G}_{0} \cap S\right)\right) \subset \mathbb{C}^{*} \cup U \text { or } \subset \mathbb{C}^{*} \cup L,
$$

where $\overline{G_{0}}$ denotes the closure of $G_{0}$ in $\mathbb{C}$. Sometimes $G_{0} \subset G^{*}$ also is chosen with the property

$$
\lambda\left(G_{0} \cup\left(\bar{G}_{0} \cap S\right)\right) \subset \mathbb{C}^{*} \cup U \cup\{1,-1\} \quad \text { or } \quad \subset \mathbb{C}^{*} \cup L \cup\{1,-1\} .
$$

With these notations pendants of Theorems 1 and 2 can be formulated for the continued fraction (7).

Theorem 6. Assume that (9) holds with $R=1$ and that the region $G_{0} \neq \varnothing$, $G_{0} \subset G^{*}$ satisfies (69). Then the following statements are true.

(a) The explicit series representations for $\widehat{A}(z), \widehat{B}(z)$, obtained from (66), (65), Corollary 1, and (22), converge absolutely and uniformly on compact subsets of $G_{0} \cup\left(\bar{G}_{0} \cap S\right)$. Hence, $\widehat{A}(z), \widehat{B}(z)$ are holomorphic on $G_{0}$ and can be extended continuously onto $G_{0} \cup\left(\bar{G}_{0} \cap S\right)$, having no common zeros there. If $\widehat{B}(z) \not \equiv$ on $G_{0}$, then the continued fraction (7) converges uniformly on compact subsets of $G_{0} \backslash\left\{z \in G_{0}: \widehat{B}(z)=0\right\}$ to $F(z)=\widehat{A}(z) / \widehat{B}(z)$. If $\widehat{B} \equiv 0$ and, hence, $\widehat{A} \neq 0$ on $G_{0}$, then (7) diverges to $\infty$ on $G_{0}$.

(b) For each fixed $z \in S$ with $\lambda(z) \neq \pm 1$ the continued fraction (7) diverges. More precisely, if with $\hat{\omega}=e^{i \theta(z)}$ from (67),

$$
M_{z}(\zeta):=2\left(\hat{\omega} C(z, \hat{\omega})-\zeta \hat{\omega}^{-1} C\left(z, \hat{\omega}^{-1}\right)\right)\left(D(z, \hat{\omega})-\zeta D\left(z, \hat{\omega}^{-1}\right)\right)^{-1}
$$

denotes a Moebius transformation (in view of $(68))$ in $\zeta$, then the nth approximant of (7) at $z$ equals $M_{z}\left(e^{i 2(n+1) \theta(z)}\right)+o(1), n \rightarrow \infty$. Hence, asymptotically all approximants of (7) lie on the image circle of the unit circle under the mapping $M_{z}(\zeta)$, which is a straight line iff $\left|D\left(z, e^{i \theta(z)}\right)\right|=\left|D\left(z, e^{-i \theta(z)}\right)\right|$.

(c) If (10) holds and if now the region $G_{0}$ has property (70), then part (a) remains valid for this $G_{0}$. Furthermore, for each $z \in S$ with $\lambda(z)= \pm 1$ (7) now converges to $F(z)= \pm 2 C(z, \pm 1) / D(z, \pm 1)$, where $C(z, \pm 1), D(z, \pm 1)$ do not vanish simultaneously.

Proof. Using (66), (65), and the fact that $\hat{\omega}(z)$ from (11) satisfies $|\hat{\omega}(z)|<1$ for $z \in G_{0}$ and $|\hat{\omega}(z)|=1, \hat{\omega}(z) \neq \pm 1$ for $z \in \overline{G_{0}} \cap S$, the proof follows by substitution of $a_{n}(z), b_{n}(z), \lambda(z), \hat{\omega}(z)$ for $a_{n}, b_{n}, \lambda, \omega$ in (2), (14), (16)(18), Lemma 1, (21), (22), (24), (25) for $R=1,(26),(27)$, Corollary 1, and Lemma 6 . This yields (a). Moreover (b) is obtained by using, in addition, (14), Corollary 2 , especially (39) and (40). In (c), $\hat{\omega}(z)= \pm 1$ is allowed for $z \in \overline{G_{0}} \cap S$ and the proof follows from (28), (29), Lemma 3, especially (30), Corollary 2(e), (14), and Lemma 6. 
For $R>1$ we define

$$
S(R):=\lambda^{-1}(E(R)) \subset G, \quad \text { where } E(R) \text { is the ellipse (6). }
$$

Theorem 7. Assume that (9) holds for some $R>1$. Let $G_{0}^{*}$ be a fixed component of $G^{*}$ and $G_{0}^{* *}$ the largest subregion of $G^{* *}$ with $G_{0}^{*} \subset G_{0}^{* *}$ such that no point of $G_{0}^{* *}$ lies above $S(R)$ but the boundary $\partial_{R} G_{0}^{* *}:=\partial G_{0}^{* *} \cap G^{* *}$ of $G_{0}^{* *}$ on $G^{* *}$ lies above $S(R)$ (see (71)).

Then the following statements are true.

(a) The explicit series representations for $\widehat{A}, \widehat{B}$ obtained from (66), (65), Corollary 1 and (22) converge absolutely and uniformly on compact subsets of $G_{0}^{* *} \cup \partial_{R} G_{0}^{* *}$. Hence, $\widehat{A}, \widehat{B}$ can be extended analytically from $G_{0}^{*}$ across $S$ into $G_{0}^{* *}$ and continuously onto $G_{0}^{* *} \cup \partial_{R} G_{0}^{* *}$ having no common zeros there.

(b) The branch points of $\hat{\omega}(z)$ in (11) also are algebraic first order branch points for the extended meromorphic function (see (7)) $F(z)=\hat{A}(z) / \hat{B}(z)$, provided $\hat{B} \not \equiv 0$ on $G_{0}^{*}$. At each $z_{0} \in S$ with $\lambda\left(z_{0}\right)= \pm 1$ of even order $\hat{A}$ and $\hat{B}$ consist of two separate holomorphic branches in a neighbourhood of $z_{0}$.

(c) If (9) holds for all $R>1$, then from each component of $G^{*} \hat{A}$ and $\hat{B}$ can be extended analytically across $S$ into the whole Riemann surface $G^{* *}$. If $\widehat{B} \not \equiv 0$, then $F$ is meromorphic on $G^{* *}$.

Proof. Using (66), (65), and the fact that now $\hat{\omega}(z)$ from (11) satisfies $|\hat{\omega}(z)|<$ 1 for $z \in G_{0}^{*}|\hat{\omega}(z)|<R^{1 / 2}$ for $z \in G_{0}^{* *}$ and $|\hat{\omega}(z)|=R^{1 / 2}$ for $z \in \partial_{R} G_{0}^{* *}$, the proof of (a) follows again by substitution of $a_{n}(z), b_{n}(z), \hat{\omega}(z)$ for $a_{n}, b_{n}, \omega$ in (16), (23), (25), Lemma 5, especially (41), Corollary 3, and Lemma 6. (b) At each $z_{0} \in S$ with $\lambda\left(z_{0}\right)= \pm 1$ of even order $\hat{\omega}(z)$ has two separate holomorphic branches in a neighbourhood of $z_{0}$.

Remark 9. Obviously an analogue of (42) or (53) easily can be formulated for $\widehat{A}, \widehat{B}$.

The next theorem generalizes Theorem 7 , since it may lead to larger subregions of $G^{* *}$ into which analytic extension of $\widehat{A}, \widehat{B}$ is possible.

Theorem 8. Suppose that (9) holds with $R=1$. Let $G_{1}^{*}$ be a fixed component of $G^{*}$. Moreover, let $G_{1}^{* *}$ be a subregion of $G^{* *}$ and $H_{1}^{* *}$ a subset of $G^{* *}$ such that $G_{1}^{*} \subsetneq G_{1}^{* *} \subset H_{1}^{* *} \subset G^{* *}$ and that

$$
\sum_{j=1}^{\infty}\left(\left|a_{j}(z)-1 / 4\right|+\left|b_{j}(z)\right|\right)|\hat{\omega}(z)|^{2 j}<\infty
$$

holds uniformly on compact subsets of $H_{1}^{* *}$, where $\hat{\omega}(z)$ from $(11)$ is assumed to be extended analytically onto $G^{* *}$ with $|\hat{\omega}(z)|<1$ for $z \in G_{1}^{*}$ and $\hat{\omega}(z) \neq \pm 1$ for $z \in H_{1}^{* *}$.

Then the explicit series representations for $\widehat{A}(z), \widehat{B}(z)$ obtained from (66), (65), Corollary 1 and (22) converge absolutely and uniformly on compact subsets of $H_{1}^{* *}$. Hence, $\widehat{A}, \widehat{B}$ can be extended analytically from $G_{1}^{*}$ across $S$ into $G_{1}^{* *}$ and continuously onto $H_{1}^{* *}$ having no common zeros there.

Proof. Let $c_{k, j}(z, \omega), S_{k, r}(z, \omega)$ be obtained from $c_{k, j}(\omega)$ in (16) and $S_{k, r}(\omega)$ in (21) by substituting $a_{n}(z), b_{n}(z)$ for $a_{n}=\left(1-v_{n}\right) / 4, b_{n}=u_{n} / 2$. Then (16) yields for $z \in H_{1}^{* *}$ and $j>k \geq-1$,

$$
\left|c_{k, j}(z, \hat{\omega}(z))\right| \leq\left|1-(\hat{\omega}(z))^{2}\right|^{-1} \gamma_{k, j}(z),
$$


where for $|\hat{\omega}(z)| \geq 1$,

$$
\begin{aligned}
\gamma_{k, j}(z):= & \left|\hat{\omega}(z) u_{j}(z)\right|\left(1+|\hat{\omega}(z)|^{2(j-k)}\right) \\
& +|\hat{\omega}(z)|^{2}\left|v_{j}(z)\right|\left(1+|\hat{\omega}(z)|^{2(j-k-1)}\right),
\end{aligned}
$$

and for $|\hat{\omega}(z)| \leq 1$ (see (24)) $\gamma_{k, j}(z):=2\left(\left|u_{j}(z)\right|+\left|v_{j}(z)\right|\right)$. Then, because of (9) with $R=1$ and (72), $\rho_{k}^{*}(z):=\sum_{j=k+1}^{\infty} \gamma_{k, j}(z)<\infty$ and $\rho_{k}^{*}(z) \searrow 0$ for $k \nearrow \infty$ hold uniformly on compact subsets of $H_{1}^{* *}$. Using the recursive definition (21) of $S_{k, r}$ one proves by induction on $r$ that, in analogy with (41) in Lemma 5 ,

$$
\left|S_{k, r}(z, \hat{\omega}(z))\right| \leq\left|1-(\hat{\omega}(z))^{2}\right|^{-r} \rho_{k}^{*}(z) \rho_{k+1}^{*}(z) \cdots \rho_{k+r-1}^{*}(z)
$$

holds for all $z \in H_{1}^{* *}, k \geq-1, r \geq 1$. Then also

$$
\sum_{r=1}^{\infty}\left|1-(\hat{\omega}(z))^{2}\right|^{-r} \rho_{k}^{*}(z) \rho_{k+1}^{*}(z) \cdots \rho_{k+r-1}^{*}(z)<\infty
$$

is valid uniformly on compact subsets of $H_{1}^{* *}$, and the assertion now follows as in the proof of Theorem 7 . That $\widehat{A}, \widehat{B}$ do not vanish simultaneously on $H_{1}^{* *}$ follows, by substitution, from (43) in Lemma 6 or from Remark 4.

\section{THE MAIN RESULTS CONCERNING GENERAL T-FRACTIONS (12)}

We now are going to apply Theorems 6 and 7 to general limit periodic $T$ fractions $T(z)$ defined in (12) and satisfying (13). For all pairs $c, d \in \mathbb{C}$ we will describe explicitly the cut $S \subset \mathbb{C}$, being the boundary of the convergence region $\mathbb{C} \backslash S$ of (12) and, if (13) holds for some $R>1$, we will derive explicit parameter representations for the boundary curves $S(R)$ up to which meromorphic extension of $T(z)$ across $S$ is possible. For partial results obtained so far we refer to $[4,5,9,11,12,13,14]$.

Remark 10. If $c=0$, then Worpitzky's Theorem (see $[6,8,15]$ ) implies that (12) converges and $T(z)$ is meromorphic on $\mathbb{C}$ (or $\mathbb{C} \backslash\{-1 / d\})$ in case $d=0$ (or $d \neq 0$ ).

Therefore, we will always assume $c \neq 0$. Next, we have to apply an equivalence transformation to (12) in order to obtain a continued fraction of type (7) to which Theorems 6 and 7 are applicable.

If $d=0$, then we can assume w.l.o.g. that $c=1 / 4$ (otherwise replace $z$ by $z / 4 c)$. In this case $(12)$ is equivalent to $(z \neq 0)$,

$$
T(z)=\frac{z^{-1 / 2}}{z^{-1 / 2}+d_{0} z^{1 / 2}}-\frac{c_{1}}{z^{-1 / 2}+d_{1} z^{1 / 2}}-\frac{c_{2}}{z^{-1 / 2}+d_{2} z^{1 / 2}}-\cdots .
$$

Observe, that the value of this continued fraction is independent of the chosen branch of $z^{1 / 2}$ and $z^{-1 / 2}:=\left(z^{1 / 2}\right)^{-1}$. We then can apply Theorems 6 and 7 to (73), if we choose a single-valued branch of $z^{1 / 2}$ for $z \in G:=\mathbb{C} \backslash L$, where $L$ is an arbitrary fixed ray from 0 to $\infty$. Except for the first partial numerator $z^{-1 / 2},(73)$ then is of type (7) with $a_{n}:=c_{n}, n \geq 1, b_{n}(z):=d_{n} z^{1 / 2}, n \geq 0$, $\lambda(z):=z^{-1 / 2}, z \in G$, such that $(9)$ is satisfied on $G$ with the same value of $R$ as in (13). 
If $d \neq 0$, then we can assume w.l.o.g. that $d=1$ (otherwise replace $z$ by $z / d)$. In this case $(12)$ is equivalent to $(z \neq 0)$,

$$
\begin{aligned}
T(z)= & \frac{(4 c z)^{-1 / 2}}{(4 c z)^{-1 / 2}+d_{0}(z / 4 c)^{1 / 2}}-\frac{c_{1} / 4 c}{(4 c z)^{-1 / 2}+d_{1}(z / 4 c)^{1 / 2}} \\
& -\frac{c_{2} / 4 c}{(4 c z)^{-1 / 2}+d_{2}(z / 4 c)^{1 / 2}}-\cdots .
\end{aligned}
$$

Again the value of this continued fraction is independent of the chosen branch of $(4 c z)^{-1 / 2}$ and $(z / 4 c)^{1 / 2}:=z(4 c z)^{-1 / 2}$. We then can apply Theorems 6 and 7 to (74), if we choose a single-valued branch of $(4 c z)^{-1 / 2}$ for $z \in G:=$ $\mathbb{C} \backslash L$, where $L$ is an arbitrary fixed ray from 0 to $\infty$. Except for the first partial numerator $(4 c z)^{-1 / 2}$ (74) then is of type (7) with $a_{n}:=c_{n} / 4 c, n \geq 1$, $b_{n}(z):=\left(d_{n}-1\right)(z / 4 c)^{1 / 2}, n \geq 0$,

$$
\lambda(z):=c^{-1 / 2}\left(z^{1 / 2}+z^{-1 / 2}\right) / 2=(z / 4 c)^{1 / 2}+(4 c z)^{-1 / 2}, \quad z \in G,
$$

such that (9) is satisfied on $G$ with the same $R$ as in (13). Observe that here $\lambda(1 / z)=\lambda(z)$ and $\lambda^{\prime}(z) \neq 0$ for $z \neq 1$. Always $-1 \in S$ holds, since $\lambda(-1)=0$.

Altogether we thus obtain the following result. If (12) satisfies (13), then all statements of Theorems 6 and 7 are valid for the special continued fractions (73) and (74). Therefore all statements of Theorems 6 and 7 concerning convergence, divergence, meromorphic extension across $S$ and continuous extension up to the boundary described by $S(R)$ also are valid for the continued fraction (12) and $T(z)$, where now $G=\mathbb{C} \backslash L$ can be replaced by $\mathbb{C}$ again. What remains to be done in the following theorems is to determine explicitly $S$ in (8) and $S(R), R>1$, in (71) for $\lambda(z)=z^{-1 / 2}$ in case $d=0, c=1 / 4$ and for $\lambda(z)=c^{-1 / 2}\left(z^{1 / 2}+z^{-1 / 2}\right) / 2$ in case $d=1, c \in \mathbb{C}, c \neq 0$.

Theorem 9 (Determination of $S$ ). Assume that (12) satisfies $\lim _{n \rightarrow \infty} c_{n}=c \in \mathbb{C}$, $c \neq 0$, and $\lim _{n \rightarrow \infty} d_{n}=d \in \mathbb{C}$. Then the following statements are true.

(a) If $d=0$ and (w.l.o.g.) $c=1 / 4$, then $S=[1, \infty) \subset \mathbb{R}^{+}$.

(b) Let $c \in \mathbb{C}$ and $d=1$ (w.l.o.g. if $d \neq 0$ ). Then always $-1 \in S=$ $\left\{\left(t c^{1 / 2}+\left(t^{2} c-1\right)^{1 / 2}\right)^{2}:-1 \leq t \leq 1\right\}$ holds. In $\left(b_{1}\right),\left(b_{2}\right),\left(b_{4}\right)$ below all square roots are $>0$.

$\left(b_{1}\right)$ If $d=1, c<0$, then

$$
S=\left[-\left((|c|+1)^{1 / 2}+|c|^{1 / 2}\right)^{2},-\left((|c|+1)^{1 / 2}-|c|^{1 / 2}\right)^{2}\right] \subset \mathbb{R}^{-} .
$$

$\left(\mathrm{b}_{2}\right)$ If $d=1,0<c<1$, then $S$ is the subarc of the unit circle which contains -1 and has the endpoints $\left(c^{1 / 2} \pm i(1-c)^{1 / 2}\right)^{2}$.

$\left(b_{3}\right)$ If $d=1, c=1$, then $S=\{z \in \mathbb{C}:|z|=1\}$.

(b) If $d=1, c>1$, then $S=I \cup\{z \in \mathbb{C}:|z|=1\}$, where $I:=\left[\left(c^{1 / 2}-(c-1)^{1 / 2}\right)^{2},\left(c^{1 / 2}+(c-1)^{1 / 2}\right)^{2}\right] \subset \mathbb{R}^{+}$with $1 \in I$.

$\left(\mathrm{b}_{5}\right)$ If $d=1, c=|c| e^{i \gamma}$ with $0<\gamma<\pi$, then $S \subset S^{\gamma}$, where $S^{\gamma}:=$ $\left\{z=r e^{i \psi}: r=r(\psi)=\sin ((\psi+\gamma) / 2) / \sin ((\psi-\gamma) / 2), \gamma<\psi<2 \pi-\gamma\right\}$ is a trigonometric spiral (being independent of $|c|)$. For $\gamma \leq \psi \leq 2 \pi-\gamma, r(\psi)$ strictly decreases from $\infty$ to $0 . S$ is the subarc of $S^{\gamma}$ which passes through -1 and has the endpoints $r\left(\psi_{0}\right) e^{i \psi_{0}}, r\left(2 \pi-\psi_{0}\right) e^{-i \psi_{0}}$, where $\cos \psi_{0}:=|c|-|c-1|$, $\gamma<\psi_{0}<\pi$ (observe ||$c|-| c-1||<1$, since $\operatorname{Im} c>0$ ). If $d=1$ and $\operatorname{Im} c<0$, then a result holds which is symmetric to the previous one. 
Proof. (a) Put $z=r e^{i \psi}, r>0, \psi \in \mathbb{R}$. Then $\lambda(z)=z^{-1 / 2}=r^{-1 / 2} e^{-i \psi / 2}$ and $z \in S$ iff $\operatorname{Im} \lambda(z)=0$ and $|\operatorname{Re} \lambda(z)| \leq 1$, i.e., iff $\sin \psi / 2=0$ and $r^{-1 / 2}|\cos \psi / 2| \leq 1$. Hence, $S=[1, \infty)$.

(b) Solving the equation $\lambda(z)=t$, i.e., $c^{-1 / 2}\left(z^{1 / 2}+z^{-1 / 2}\right) / 2=t$, for $z$ yields $z=z(t)=\left(t c^{1 / 2} \pm\left(t^{2} c-1\right)^{1 / 2}\right)^{2}=c\left(t \pm\left(t^{2}-c^{-1}\right)^{1 / 2}\right)^{2}$. Then $S=$ $\{z(t):-1 \leq t \leq 1\}$ and $z(0)=-1$.

$\left(b_{1}\right)$ If $c<0$, then $z(t)=-\left(t|c|^{1 / 2} \pm\left(t^{2}|c|+1\right)^{1 / 2}\right)^{2}$. Hence, $S$ is the closed interval $\subset \mathbb{R}^{-}$with endpoints $z( \pm 1)=-\left(|c|^{1 / 2} \pm(|c|+1)^{1 / 2}\right)^{2}$.

Next, $c>0$ yields $z(t)=\left(t c^{1 / 2} \pm i\left(1-t^{2} c\right)^{1 / 2}\right)^{2},-1 \leq t \leq 1$.

$\left(b_{2}\right)$ If $0<c<1$, then $S$ is an arc of the unit circle with endpoints $z( \pm 1)$.

$\left(b_{3}\right)$ If $c=1$, then $S$ is the whole unit circle, since then $z( \pm 1)=1$.

$\left(b_{4}\right)$ If $c>1$, then $z(t)$ describes the whole unit circle for $-c^{-1 / 2} \leq t \leq$ $c^{-1 / 2}<1\left(z\left( \pm c^{-1 / 2}\right)=1\right)$, and for $c^{-1 / 2} \leq|t| \leq 1, z(t)=\left(t c^{1 / 2} \pm\left(t^{2} c-1\right)^{1 / 2}\right)^{2}$ describes the interval $\subset \mathbb{R}^{+}$which intersects the unit circle in $z=1$ and has endpoints $z( \pm 1)$.

$\left(\mathrm{b}_{5}\right)$ In this case we again put $z=r e^{i \psi}, r>0, \psi \in \mathbb{R}$, and then determine $S$ by substituting

$$
\lambda(z)=c^{-1 / 2}\left(z^{1 / 2}+z^{-1 / 2}\right) / 2= \pm\left(r^{1 / 2} e^{i(\psi-\gamma) / 2}+r^{-1 / 2} e^{-i(\psi+\gamma) / 2}\right) / 2|c|^{1 / 2}
$$

in the conditions $\operatorname{Im} \lambda(z)=0,|\operatorname{Re} \lambda(z)| \leq 1$. This yields

$$
\begin{aligned}
r & =r(\psi)=\sin ((\psi+\gamma) / 2) / \sin ((\psi-\gamma) / 2) \\
& =\sin \gamma(\operatorname{ctg} \gamma+\operatorname{ctg}((\psi-\gamma) / 2))>0
\end{aligned}
$$

and $r^{\prime}(\psi)<0$ for $\gamma<\psi<2 \pi-\gamma$. Next, $(\operatorname{Re} \lambda(z))^{2} \leq 1$ is equivalent with

$$
\begin{aligned}
& r \cos ^{2}((\psi-\gamma) / 2)+2 \cos ((\psi-\gamma) / 2) \cos ((\psi+\gamma) / 2) \\
& \quad+r^{-1} \cos ^{2}((\psi+\gamma) / 2) \leq 4|c| .
\end{aligned}
$$

Here we substitute the value $r=r(\psi)$ obtained above and multiply by

$$
\sin ((\psi+\gamma) / 2) \sin ((\psi-\gamma) / 2)>0 .
$$

Then we obtain equivalently

$$
\begin{aligned}
& (\sin ((\psi+\gamma) / 2) \cos ((\psi-\gamma) / 2)+\sin ((\psi-\gamma) / 2) \cos ((\psi+\gamma) / 2))^{2} \\
& \quad \leq 4|c| \sin ((\psi+\gamma) / 2) \sin ((\psi-\gamma) / 2)
\end{aligned}
$$

or $\sin ^{2} \psi \leq 2|c|(\cos \gamma-\cos \psi)$. Adding $\cos ^{2} \psi+|c|^{2}-2|c| \cos \gamma$ on both sides yields $1-2|c| \cos \gamma+|c|^{2} \leq \cos ^{2} \psi-2|c| \cos \psi+|c|^{2}$, or $|c-1|^{2} \leq(\cos \psi-|c|)^{2}$, or $|c-1| \leq|\cos \psi-| c||$. This is equivalent with $\cos \psi-|c| \leq-|c-1|$, since $\cos \psi-|c| \geq|c-1|$ cannot hold because of $|c|+|c-1|>1$ for $\operatorname{Im} c \neq 0$. Hence, $S$ is the subarc of $S^{\gamma}$ which is described by $\cos \psi \leq|c|-|c-1|$ and $\gamma<\psi<2 \pi-\gamma$, or by $\psi_{0} \leq \psi \leq 2 \pi-\psi_{0}$, where $\psi_{0}$ is the unique solution of $\cos \psi_{0}=|c|-|c-1|$ and $\gamma<\psi_{0}<\pi$ (always ||$c|-| c-1||<1$ holds for $\operatorname{Im} c \neq 0)$.

For $R>1$ we now define

$$
\begin{aligned}
& a:=\left(R+R^{-1}\right) / 2, b:=\left(R-R^{-1}\right) / 2, \text { implying } a>1, b>0, \\
& \text { and } \left.a^{2}-b^{2}=1 \text { (see }(6)\right) .
\end{aligned}
$$

Theorem 10 (Determination $S(R)$ ). Assume that (12) satisfies (13) for some $R>1$. Then the following statements are true. 
( $\alpha$ ) If $d=0$ and $c=1 / 4$, then (see (75))

$S(R)=\left\{z=r e^{i \psi}: r=r(\psi)=2(a-\cos \psi) / b^{2}, 0 \leq \psi \leq 2 \pi\right\}$ and $r^{\prime}(\psi)>0$, for $0<\psi<\pi$. For large $R S(R)$ is almost a circle of radius $4 / R$ around 0 . The endpoints of $S=[1, \infty)$ are first order algebraic branch points for the extended meromorphic function $T(z)$, provided $T \not \equiv \infty$.

( $\beta)$ If $d=1$ and $c=|c| e^{i \gamma} \neq 0, \gamma \in \mathbb{R}$, then $S(R)$ consists of 2 curves given by

$$
S_{ \pm}(R):=\left\{z=r_{ \pm} e^{i \psi}: r_{ \pm}=r_{ \pm}(\psi)=P_{ \pm}(\psi) / Q(\psi), \psi_{1} \leq \psi \leq 2 \pi-\psi_{1}\right\},
$$

where $(\operatorname{see}(75)) Q(\psi):=2|c|(a-\cos (\psi-\gamma))>0$ and, with $p:=a|c|+|c-1|>1$, $q:=a|c|-|c-1|>-1$,

$P_{ \pm}(\psi):=\sin ^{2} \psi+\left(b|c| \pm((p-\cos \psi)(q-\cos \psi))^{1 / 2}\right)^{2}, \quad \psi_{1} \leq \psi \leq 2 \pi-\psi_{1}$.

If $q \geq 1$, then $\psi_{1}:=0$ and if $q<1$, then $\psi_{1}$ denotes the unique solution of $\cos \psi_{1}=q, 0<\psi_{1}<\pi$.

Always $r_{+}(\psi)>r_{-}(\psi)>0$ holds for $\psi_{1}<\psi<2 \pi-\psi_{1}$. 0 .

If $q<1$, then $0<\psi_{1}<\pi, r_{+}\left(\psi_{1}\right)=r_{-}\left(\psi_{1}\right)>0, r_{+}\left(2 \pi-\psi_{1}\right)=r_{-}\left(2 \pi-\psi_{1}\right)>$

If $q=1$, then $\psi_{1}=0, r_{+}(0)=r_{+}(2 \pi)=r_{-}(0)=r_{-}(2 \pi)>0$.

If $q>1$, then $\psi_{1}=0, r_{+}(0)=r_{+}(2 \pi)>r_{-}(0)=r_{-}(2 \pi)>0$.

For large $R, S_{+}(R)$ and $S_{-}(R)$ are almost circles of radius $|c| R$ and $1 /|c| R$ respectively. If $c=1$, then $r_{+}(\psi)=R$ and $r_{-}(\psi)=1 / R$ for $0 \leq \psi \leq 2 \pi$.

Always $S$ (see Theorem $9(\mathrm{~b})$ ) lies in the region between $S_{+}(R)$ and $S_{-}(R)$. For $c \neq 1$ the endpoints $\left(c^{1 / 2} \pm(c-1)^{1 / 2}\right)^{2}$ of $S$ are first order algebraic branch points for the extended meromorphic function $T(z)$ and for $c \geq 1$ (see Theorem $\left.9\left(\mathrm{~b}_{3}\right),\left(\mathrm{b}_{4}\right)\right) \quad 1 \in S$ holds with $\lambda(1)= \pm 1$ of order two (see Remark 8 ) and the extended function $T(z)$ consists of two separate meromorphic branches in a neighbourhood of $z=1$, provided $T \not \equiv \infty$.

Proof. Put $z=r e^{i \psi}, r=r(\psi)>0, \psi \in \mathbb{R}$. By (71), $z \in S(R)$ holds iff $\lambda(z) \in E(R)$ in $(6)$.

( $\alpha$ ) Substituting $\lambda(z)=z^{-1 / 2}=r^{-1 / 2} e^{-i \psi / 2}$ for $\lambda$ in (6) yields the linear equation for $r$ :

$$
\left(R-2+R^{-1}\right) \cos ^{2}(\psi / 2)+\left(R+2+R^{-1}\right) \sin ^{2}(\psi / 2)=\left(R-R^{-1}\right)^{2} r / 4,
$$

from which the assertion follows immediately in view of (75).

( $\beta$ ) We substitute

$$
\lambda(z)=c^{-1 / 2}\left(z^{1 / 2}+z^{-1 / 2}\right) / 2= \pm\left(r^{1 / 2} e^{i(\psi-\gamma) / 2}+r^{-1 / 2} e^{-i(\psi+\gamma) / 2}\right) / 2|c|^{1 / 2}
$$

for $\lambda$ in (6) and obtain

$$
\begin{aligned}
& \left(r^{1 / 2} \cos ((\psi-\gamma) / 2)-r^{-1 / 2} \cos ((\psi+\gamma) / 2)\right)^{2}\left(R-2+R^{-1}\right) \\
& \quad+\left(r^{1 / 2} \sin ((\psi-\gamma) / 2)-r^{-1 / 2} \sin ((\psi+\gamma) / 2)\right)^{2}\left(R+2+R^{-1}\right)=|c|\left(R-R^{-1}\right)^{2} .
\end{aligned}
$$

This yields the quadratic equation for $r: r^{2}-2 r u+v=0$ with solutions $r_{ \pm}(\psi)=$ $u(\psi) \pm\left(u^{2}(\psi)-v(\psi)\right)^{1 / 2}$, where with (75)

$$
\begin{aligned}
& u(\psi):=\left(\cos \gamma-a \cos \psi+|c| b^{2}\right) /(a-\cos (\psi-\gamma)), \\
& v(\psi):=(a-\cos (\psi+\gamma)) /(a-\cos (\psi-\gamma)) .
\end{aligned}
$$


Then $u^{2}-v=b^{2}\left((a|c|-\cos \psi)^{2}-|c-1|^{2}\right) /(a-\cos (\psi-\gamma))^{2}$ and the numerator of $u$ can be written as

$$
\left((a|c|-\cos \psi)^{2}-|c-1|^{2}+\sin ^{2} \psi+|c|^{2} b^{2}\right) / 2|c| .
$$

Therefore, $r_{ \pm}(\psi)=P_{ \pm}(\psi) / Q(\psi)$ holds with $Q(\psi):=2|c|(a-\cos (\psi-\gamma))$ and

$$
\begin{aligned}
P_{ \pm}(\psi):= & (a|c|-\cos \psi)^{2}-|c-1|^{2}+\sin ^{2} \psi+b^{2}|c|^{2} \\
& \pm 2 b|c|\left((a|c|-\cos \psi)^{2}-|c-1|^{2}\right)^{1 / 2} \\
= & \sin ^{2} \psi+\left(b|c| \pm\left((a|c|-\cos \psi)^{2}-|c-1|^{2}\right)^{1 / 2}\right)^{2} .
\end{aligned}
$$

This and $(a|c|-\cos \psi)^{2}-|c-1|^{2}=(p-\cos \psi)(q-\cos \psi)$ yield the formula for $P_{ \pm}(\psi)$ as asserted in part $(\beta)$, where $p:=a|c|+|c-1|>|c|+|c-1| \geq 1$, since $a>1$, and $q:=a|c|-|c-1|>-1$, since $q+|c-1|+1=a|c|+1>|c|+1 \geq|c-1|$.

Next, we observe that $P_{ \pm}(\pi)>0$ and, provided $q \geq 1, P_{ \pm}(0)>0$, since otherwise by means of (75) $(a|c| \pm 1)^{2}-|c-1|^{2}=b^{2}|c|^{2}$ or $\pm \operatorname{Re} c=a|c|$ would follow, which is impossible because of $a>1$. Since generally $(x+y)^{2}>(x-y)^{2}$ holds for $x, y>0$, the rest of the assertion of $(\beta)$ follows from the above explicit representation of $P_{ \pm}(\psi)$ as sum of 2 squares of real expressions.

Remark 11. If in Theorem $10(\beta)$ we solve the equation $\lambda(z)=t, t \in E=$ $E(R)$ (see (6)) for $z$, we obtain the parameter representations

$$
z_{ \pm}(t)=\left(t c^{1 / 2} \pm\left(t c^{1 / 2}-1\right)^{1 / 2}\left(t c^{1 / 2}+1\right)^{1 / 2}\right)^{2}, \quad t \in E
$$

for the two curves $S_{ \pm}(R)$. Furthermore, it follows easily, that in Theorem $10(\beta), q>1, q=1$, or $q<1$ holds iff $\pm c^{-1 / 2}$ are inside $E$, on $E$ or outside $E$.

Remark 12. If Theorem 8 is applied to the continued fractions (73) and (74), more general meromorphic extension results concerning $T(z)$ in (12) may be obtained.

We conclude our considerations with the following example. Assume that in (12) $c_{n}=d_{n}=1$ holds for $n \geq 2$. Then $c=d=1$ and Theorem $9\left(\mathrm{~b}_{3}\right)$ is applicable. In this special case it is easy to compute $T(z)$ and its approximants directly for $|z|<1$ and $|z|>1$, being the 2 components of $G^{*}=G \backslash S$, where $G=\mathbb{C}$ and $S=\{z \in \mathbb{C}:|z|=1\}$. But, as an illustration, we want to compute $T(z)$ for $|z|<1$ and $|z|>1$ and its meromorphic extension across $|z|=1$ by applying Theorem 7 to (74), which is equivalent to (12). To be precise one should use a single-valued branch of $z^{1 / 2}$ and $z^{-1 / 2}=\left(z^{1 / 2}\right)^{-1}$ on $\mathbb{C} \backslash L$, where $L$ is an arbitrary fixed ray from 0 to $\infty$, which at the end of our consideration can be deleted again, since the value of $T(z)$ is independent of the chosen branch of $z^{1 / 2}$. Then in (74), $b_{n}=0, a_{n}=1 / 4$ for $n \geq 2$, and hence $v_{n}=1-4 a_{n}=0, u_{n}=2 b_{n}=0$ for $n \geq 2$. Moreover, $v_{1}=1-c_{1}$, $u_{n}(z)=\left(d_{n}-1\right) z^{1 / 2}$ for $n=0,1$ and $\lambda(z)=\left(z^{1 / 2}+z^{-1 / 2}\right) / 2$.

In this special case $(21),(22)$, and Corollary 1 yield

$$
\left\{\begin{aligned}
C(\omega) & =1+c_{0,1}=1+\omega u_{1}, \\
D(\omega) & =1+c_{-1,0}+c_{-1,1}+c_{-1,0} c_{0,1} \\
& =1+\omega u_{0}+\left(\omega u_{1}(1+w)+w v_{1}\right)+w u_{0} u_{1},
\end{aligned}\right.
$$

and (11) yields $\hat{\omega}(z)=z^{1 / 2}$ for $|z|<1$, and $\hat{\omega}(z)=z^{-1 / 2}$ for $|z|>1$, implying $|\hat{\omega}(z)|<1$ in both cases. 
For $|z|<1$ we then obtain from (66) and (76)

$$
\widehat{A}(z)=2 z^{1 / 2}\left(1+\left(d_{1}-1\right) z\right) \not \equiv 0
$$

and

$$
\widehat{B}(z)=\left(1+d_{0} z\right)\left(1+\left(d_{1}-1\right) z\right)-c_{1} z \not \equiv 0,
$$

where $\widehat{A}(z), \widehat{B}(z)$ have no common zeros. Hence, (see (74))

$$
\begin{aligned}
T(z) & =(4 z)^{-1 / 2} \widehat{A}(z) / \widehat{B}(z) \\
& =\left(1+\left(d_{1}-1\right) z\right) /\left(\left(1+d_{0} z\right)\left(1+\left(d_{1}-1\right) z\right)-c_{1} z\right) \text { for }|z|<1 .
\end{aligned}
$$

In accordance with Theorem $7(\mathrm{c}), T(z)$ can be extended meromorphically from $|z|<1$ onto $\mathbb{C}$.

For $|z|>1,(66),(76)$, and $\hat{\omega}(z)=z^{-1 / 2}$ yield $\widehat{A}(z)=2 z^{-1 / 2} d_{1}, \widehat{B}(z)=$ $d_{1}\left(d_{0}+z^{-1}\right)-c_{1} z^{-1}$. If here $d_{1}=0$, then $\widehat{A}(z) \equiv 0$ and $\widehat{B}(z)=-c_{1} z^{-1} \neq 0$ for $|z|>1$. If $d_{0}=0$ and $d_{1}=c_{1} \neq 0$, then $\widehat{B}(z) \equiv 0$ and $\widehat{A}(z) \neq 0$ for $|z|>1$. If $\widehat{B}(z) \not \equiv 0$, then (see equation (74)) $T(z)=(4 z)^{-1 / 2} \widehat{A}(z) / \widehat{B}(z)=$ $d_{1} /\left(d_{1}\left(1+d_{0} z\right)-c_{1}\right)$ for $|z|>1$ and in accordance with Theorem $7(\mathrm{c}) T(z)$ can be extended meromorphically from $|z|>1$ onto $\mathbb{C}$.

\section{REFERENCES}

1. R. Askey and M. Ismail, Recurrence relations, continued fractions and orthogonal polynomials, Mem. Amer. Math. Soc., no. 300, 1984.

2. G. A. Baker, Jr. and P. Graves-Morris, Padé approximants, part I: Basic theory, Encyclopedia of Math. and its Applications, No. 13, Addison-Wesley, Reading, Mass., 1981.

3. J. Gill, Enhancing the convergence region of a sequence of bilinear transformations, Math. Scand. 43 (1978), 74-80.

4. L. Jacobsen, Functions defined by continued fractions meromorphic continuation, Rocky Mountain J. Math. 15 (1985), 685-703.

5. __ Approximants for functions represented by limit periodic continued fractions, Constructive Theory of Functions (Varna, 1987), Bulgar. Acad. Sci., Sofia, 1988, pp. 242-250.

6. W. B. Jones and W. J. Thron, Continued fractions, analytic theory and applications, Encyclopedia of Math. and its Applications, No. 11, Addison-Wesley, Reading, Mass., 1980.

7. P. G. Nevai, Orthogonal polynomials, Mem. Amer. Math. Soc., no. 213, 1979.

8. O. Perron, Die Lehre von den Kettenbrüchen, Bd. 2, Teubner, Stuttgart, 1957.

9. H.-J. Runckel, Continuity on the boundary and analytic continuation of continued fractions, Math. Z. 148 (1976), 189-205.

10. __ Zeros of complex orthogonal polynomials, Internat. Sympos. on Orthogonal Polynomials and their Applications for the 150th Anniversary of E. N. Laguerre, (Bar-Le-Duc, France, 1984), Lecture Notes in Math., vol. 1171, Springer-Verlag, Berlin, 1985, pp. 278282.

11. Meromorphic extension of analytic continued fractions across the line of nonconvergence, Rocky Mountain J. Math. 21 (1991), 539-556.

12. H. Schlierf, Analytische Fortsetzung von limitärperiodischen und (2, 1)-limitärperiodischen $\delta$-Kettenbrüchen sowie Integral-darstellungen für spezielle $\delta$-Kettenbrüche, Dissertation, University of Ulm, 1988.

13. W. J. Thron and $\mathbf{H}$. Waadeland, Accelerating convergence of limit periodic continued fractions $K\left(a_{n} / 1\right)$, Numer. Math. 34 (1980), 155-170. 
14. _ Analytic continuation of functions defined by means of continued fractions, Math. Scand. 47 (1980), 72-90.

15. H. S. Wall, Analytic theory of continued fractions, Van Nostrand, New York, 1948.

Abteilung Mathematik IV, Universität Ulm, Oberer Eselsberg, D-7900 Ulm, Germany E-mail address: hof@dulruu.51.bitnet 